\title{
A Review of Organochlorine Contaminants in Nearshore Marine Mammal Predators
}

Amy Green* and Shawn Larson

Seattle Aquarium, 1483 Alaskan Way, Seattle, WA 98101, USA

\begin{abstract}
Nearshore vertebrate predators such as marine mammals and otters are apex predators, and as such, act both as drivers of the ecosystems in which they live and as sentinels of environmental health. As apex predators, these wild animals at the top of the food chain often bioaccumalate persistent organic pollutants (termed POPs) widespread throughout the environment. Organochlorines are a type of POP that tends to be lipophilic and hydrophobic which accumulate in the fatty tissues of marine mammals and other vertebrate predators over time. There has been growing concern about these POPs in the marine environment and within wildlife, as they can potentially cause health problems. Since the 1950s there have been 81,000 papers published on organochlorines in nearshore predators and potential negative effects to the environment, wildlife and humans. Here, we review organochlorines reported in the tissues of marine mammals and other nearshore vertebrate predators since 1995 . We focus on five organochlorines that have been studied the most within nearshore vertebrate predators: 2,2-bis-(p-chlorophenyl)-1,1,1-trichloroethane or dichlorodiphenyltrichloroethane (DDT), polychlorinated biphenyls (PCBs), polybrominated diphenyl ethers (PBDEs), toxaphene, and polychlorinated naphthalnese (PCNs).
\end{abstract}

Keywords: Organochlorine; Contaminants; Persistent organic pollutants; Nearshore vertebrate marine mammal predators; Wildlife

\section{Introduction}

Life history characteristics such as long life spans and limited home ranges make marine mammals living indicators and sentinels of environmental health. By monitoring wildlife health, we can infer the relative health of the environment in which they live and use data from these living sentinels to investigate shifts and changes in the ecosystem. Environmental contaminants from man-made chemicals are found to be widespread, many of which have unknown toxic effects at the ecosystem, population and individual level. Some environmental contaminants are short lived and cause acute rather than chronic damage. Others are persistent in the environment and may cause chronic environmental and wildlife health problems long after they are manufactured and even banned. In addition, some may also accumulate, increasing over time in individuals (bioaccumulation) or in orders of magnitude with each trophic level (biomagnification). The most damaging to wildlife, and in turn their ecosystem, are those contaminants that are persistent and bioaccumulate.

Contaminants may enter the environment through air, soil, and water. They are easily spread throughout the nearshore marine environment primarily due to the proximity of dense human populations that facilitate the transfer of contaminants from land to water via runoff. Contaminants may also travel farther offshore from their origins via atmospheric deposition through atmospheric currents and precipitation. Because of their widespread and persistent nature, there are rising concerns on the effects of environmental contaminants on ecosystems. Organochlorines are of particular concern, as they bioaccumulate through the food chain, are lipophilic, hydrophobic and persistent in the environment. In the past two decades there has been increasing concern about the effects of persistent and bioaccumulated toxicants in long lived apex predators in the marine environment. These apex predators include nearshore vertebrate and marine mammals. Aquatic organisms are good bio-indicators of environmental pollution because they concentrate bioaccumulative pollutants in their bodies from water and sediment, in addition to uptake from diet. There are numerous publications on marine mammal toxicology, but vary widely in methods used and variables measured. Determination of the potential impacts to marine animal health is still not completely understood. Differences in species, age, diet, geographical location, health, and proximity to environmental contaminants all can affect the chemical load an individual carries. In this review, we have compiled and reviewed nearshore marine mammal toxicology literature from the last 25 years (1990's to present), to establish the current state of knowledge and priorities for future research.

\section{Toxins in nearshore vertebrate predators}

Species included in this review were limited to marine nearshore vertebrate predators including pinnipeds (sea lions, fur seals, and true seals), cetaceans (porpoises, dolphins, and other odontocene or toothed whales), polar bears and mustelids (sea otters and river otters). We are defining nearshore inhabitants as species that spend most or all of their life near coastal environments. Orcinus orca, or killer whales, are the most widely ranging mammal on this list, but are also commonly found in nearshore areas [1].

Contaminants were limited to organochlorines including 2,2-bis-(p-chlorophenyl)-1,1,1-trichloroethane or dichlorodiphenyltrichloroethane (DDT), polychlorinated biphenyls (PCBs), polybrominated diphenyl ethers (PBDEs), chlordanes (CHL), hexachlorocyclohexan $(\mathrm{HCH})$, polychlorinated dibenzo- $p$ dioxins (PCDDs), polychlorinated dibenzofurans (PCDFs), mirex, toxaphene, hexachlorobenzene (HCBs), Dieldrin, and polychlorinated naphthalnese (PCNs). All organochlorines are POPs, are persistent and bioaccumulate. The organochlorines that have been studied the most

*Corresponding author: Amy Green, Seattle Aquarium, 1483 Alaskan Way, Seattle, WA 98101, USA, Tel: 2066936242; E-mail: a.green@seattleaquarium.org

Received March 18, 2016; Accepted May 02, 2016; Published May 10, 2016

Citation: Green A, Larson S (2016) A Review of Organochlorine Contaminants in Nearshore Marine Mammal Predators. J Environ Anal Toxicol 6: 370 doi:10.4172/2161-0525.1000370

Copyright: ( 2016 Green A, et al. This is an open-access article distributed under the terms of the Creative Commons Attribution License, which permits unrestricted use, distribution, and reproduction in any medium, provided the original author and source are credited. 
within nearshore vertebrate predators include: DDT, PCBs, PBDEs, Toxaphene and PCNs, and are the main focus of this review.

After adhering to these definitions of marine nearshore vertebrate predators and limiting the toxins reviewed, a total of 47 papers and 36 species were utilized for this review (Table 1). Of the total species, there were 14 different pinnipeds, 19 cetaceans, two mustelid and one polar bear species. Of the total papers, 21 studied pinnipeds, 21 studied cetaceans, two studied mustelids and four focused on polar bears. The number of publications on toxins in marine mammals has increased dramatically in the last 25 years starting from zero in 1950 to 81,000 through 2016 (Figure 1). Sampling of toxins and analytical methods varied between papers making comparisons between studies and general conclusions difficult. Tissue samples include blubber, liver, muscle, blood and skin biopsy (Table 1). Analytical tools include high performance mass spectrometry/liquid chromatography or mass spectrometry/gas chromatography. Geographical locations varied, with most research conducted in the United States, followed by Canada and Norway (Figure 2), with the vast majority of the research on marine mammal toxicology conducted in the northern hemisphere (Figure 3).

Organochlorines are the most heavily studied environmental contaminant [2]. They are a group of industrial and agricultural compounds that are lipophilic and hydrophobic [3]. They were designed for chemical stability and thus persist in the environment. These contaminants enter the marine environment through municipal and industrial wastewater outfalls, landfill leachate, and atmospheric deposition [4].

\section{Dichlorodiphenyltrichloroethane (DDT):}

Dichlorodiphenyltrichloroethane (DDT) and its metabolites are the most widely described organochlorine contaminant. It has been used worldwide as a potent chemical pesticide. It is banned in most developed countries, but is still used in other parts of the world. It is a neurotoxin and can cause pathological changes to the liver and reproductive system in animals [3]. Even though it has been in widespread use worldwide, there are relatively few values for levels found within nearshore vertebrate predators presumably because DDT was banned for use in the United States in the 1970s. The highest DDT level found in nearshore marine mammals was measured in transient killer whale (Orcinus orca) blubber from Kenai Fjords/Prince William Sound with a very high value of $320,000 \mathrm{ng} / \mathrm{g}$ lipids [5]. The highest pinniped value was measured in Antarctic fur seal (Arctocephalus gazella) blubber at a value of $168 \mathrm{ng} / \mathrm{g}$ lipid weight [6]. In Nearshore vertebrate predators such as the Eurasian river otter (Lutra lutra) was found to have high values of 3,859 ng/g liver in England and Wales [7]. Lastly eastern Greenland polar bear (Ursus martitimus) blubber was found to have a moderate value of $559 \mathrm{ng} / \mathrm{g}$ lipid weight [8].

Polychlorinated biphenyls (PCBs): Polychlorinated biphenyls (PCBs) include 209 congeners with a multitude of uses including transformers, plastics and inks [2]. Blubber concentrations have been reported for a variety of marine mammals worldwide [3]. Manufacturing of PCBs occurred from 1929 through the late 1970s [9] but was halted in most industrial nations in the 1970s-1980s [3]. Despite this, as machinery systems leak, degrade or are disposed of, there is an expectation that levels in the environment will continue to increase [3]. Dose response relationships for many of the congeners have not been well established [9]. The highest PCB values reported within marine mammals was $18,135 \mu \mathrm{g} / \mathrm{kg}$ within Puget Sound harbor seal (Phoca vitulina) blubber [10]. Of the cetaceans, transient Kenai Fjords/Prince William Sound killer whale blubber had the highest value of 230,000 ng/g [5]. While Eurasian river otter liver from England and Wales had an average value of 12,928 ng/g liver tissue [7], and Polar bear blubber from East Greenland had 9,100 ng/g tissue [8].

Polybrominated diphenyl ethers (PBDEs): Polybrominated diphenyl ethers (PBDEs) are compounds that have been widely used as flame retardants in textiles, furniture, upholstery, plastics and electronics [9]. Two of the three commercial forms (penta and octa) were removed from the European (1998) and North American (2004) marketplaces. Deca-BDE remains on the market in both countries [9]. Although most compounds have been banned, it has been rapidly accumulating in the environment since its introduction in the 1970's. Levels reached their peak in 2001 at 67,000 tons emitted. Their toxic effects persist and they are thought to be neurotoxic and hormone disruptors [11-13]. Concentrations in human blood and tissue have increased exponentially almost 100-fold since the 1970s, doubling about every five years $[14,15]$. Sources of contamination into the environment occur at production of the product and throughout the life of the product as it degrades. Since 2001 Asia has been the major producer of PBDEs and most come from e-recycling and waste that is often collected in developed countries and sent to developing countries for processing. PBDEs are then released into air, sediment, sewage sludge and water in the environment.

These compounds have been documented in many marine mammals. The presence of PBDEs was first reported in archived northern fur seal (Callorhinus ursinus) samples from the Pacific coast of Japan between 1972 and 1998 [16]. A total of eight congeners of dito hepta-BDEs were detected in all the samples, with concentrations ranging from 0.33 to $100 \mathrm{ng} / \mathrm{g}$ lipid weight in 1972 and 1994, with average concentration increasing about 150-fold from 1972 to 1994 However, the levels decreased by about $50 \%$ in 1998 which coincided with the withdrawal of penta-BDEs from use in Japan.

Ramu et al. measured the levels, profiles, and distribution of PBDEs in small cetaceans from Hong Kong waters. They analyzed ten targeted PBDE congeners in the blubber, liver and kidney of IndoPacific humpback dolphins (Sousa chinensis) and finless porpoises (Neophocaena phocaenoides) that were stranded in Hong Kong coastal waters between 1995 and 2001. The total concentrations of PBDEs ranged from 230 to $980 \mathrm{ng} / \mathrm{g}$ lipid weight in the blubber of finless porpoises and from 280 to $6000 \mathrm{ng} / \mathrm{g}$ lipid weight in the blubber of humpback dolphins. Recently, the geographical distribution of PBDEs in small cetaceans from Asian waters ranged from 6.0 to $6000 \mathrm{ng} / \mathrm{g}$ lipid weight. In contrast levels within human blood taken in Chinese workers ranged from 8.61 to $46.05 \mathrm{ng} / \mathrm{g}$ of lipid weight, with an average value of $19.33 \mathrm{ng} / \mathrm{g}$ lipid weight [17].

The highest PBDE value in pinnipeds was reported from harbor seal (Phoca vitulina concolor) liver from the Northwest Atlantic Ocean at a value of 2,670 ng/g [18]. In cetaceans, southern resident killer whale blubber had between 1,200-15,000 ng/g of PBDEs [19]. Two mustelid species, California sea otter (Enhydra lutris nereis) and the Eurasian river otter, were also tested and were found to have levels of 2,170 ng/g [20] and 3,242 ng/g [7] within livers respectively. Finally polar bears from East Greenland had values of 68-75 ng/g blubber [21].

Toxaphene: Toxaphene is an organochlorine insecticide and is deposited all over the world via atmospheric transport. It has even been found in arctic marine mammal blubber far from its targeted use [2]. Toxaphene was primarily used in the 50 s through 70 s, and was banned in the US in 1982. It was the most widely used pesticide before restrictions for its use were put in place [3]. Gues et al. suggests 
Citation: Green A, Larson S (2016) A Review of Organochlorine Contaminants in Nearshore Marine Mammal Predators. J Environ Anal Toxicol 6: 370. doi:10.4172/2161-0525.1000370

Page 3 of 14

Table 1: Summary of selected organochlorine residue surveys in pinnipeds, cetaceans, mustelids and polar bears from 1991-2012. Contaminant concentration includes mean or range (ng/g lipid weight, unless otherwise stated) [6-71]

\begin{tabular}{|c|c|c|c|c|c|c|c|}
\hline Reference & Year & Location & Species & Sample Type & $\mathbf{N}$ & Chemical compound & $\begin{array}{l}\text { Amount (average or range lipid } \\
\text { weight until otherwise stated) }\end{array}$ \\
\hline [39] & $\begin{array}{l}1991- \\
1997\end{array}$ & California & $\begin{array}{l}\text { California sea } \\
\text { lion (Zalophus } \\
\text { californianus) }\end{array}$ & $\begin{array}{l}\text { Blubber, } \\
\text { Liver }\end{array}$ & 15 & $\begin{array}{l}\text { DDTs, } \\
\text { PCBs, } \\
\text { CHL, } \\
\mathrm{HCH}\end{array}$ & $\begin{array}{c}\text { Blubber: } \\
\text { DDT: } 13-2,900 \mu \mathrm{g} / \mathrm{g} \\
\text { PCBs: } 7.2-1,300 \mu \mathrm{g} / \mathrm{g} \\
\text { Chlordanes: } 0.51-86 \mu \mathrm{g} / \mathrm{g} \\
\text { HCH: } 0.15-7.0 \mu \mathrm{g} / \mathrm{g} \\
\text { Liver: } \\
\text { DDT: } 12-970 \mu \mathrm{g} / \mathrm{g} \\
\text { PCBs: } 11-410 \mu \mathrm{g} / \mathrm{g} \\
\text { Chlordanes: } 0.44-37 \mu \mathrm{g} / \mathrm{g} \\
\text { HCH: } 0.17-4.5 \mu \mathrm{g} / \mathrm{g}\end{array}$ \\
\hline [39] & $\begin{array}{l}1991- \\
1997\end{array}$ & California & $\begin{array}{c}\text { Harbor seal (Phoca } \\
\text { vitulina) }\end{array}$ & Liver & 10 & $\begin{array}{l}\text { DDTs, } \\
\text { PCBs }\end{array}$ & $\begin{array}{c}\text { DDT: } 2.8-85 \mathrm{ng} / \mathrm{g} \\
\text { PCBs: } 5-350 \mu \mathrm{g} / \mathrm{g} \\
\text { Chlordanes: } 0.11-4 \mu \mathrm{g} / \mathrm{g} \\
\mathrm{HCH}: \text { Not detectable-. } 040 \mu \mathrm{g} / \mathrm{g}\end{array}$ \\
\hline [39] & $\begin{array}{l}1991- \\
1997\end{array}$ & California & $\begin{array}{l}\text { Elephant seal } \\
\text { (Mirounga } \\
\text { augustirostris) }\end{array}$ & $\begin{array}{l}\text { Blubber, } \\
\text { Liver }\end{array}$ & 6 & $\begin{array}{l}\text { DDTs, } \\
\text { PCBs }\end{array}$ & $\begin{array}{c}\text { Blubber: } \\
\text { DDT: } 8.3-110 \mu \mathrm{g} / \mathrm{g} \\
\text { PCBs: } 5.0-58 \mu \mathrm{g} / \mathrm{g} \\
\text { Chlordanes: } 0.83-8.6 \mu \mathrm{g} / \mathrm{g} \\
\text { HCH: } 0.12-0.90 \mu \mathrm{g} / \mathrm{g} \\
\text { Liver: } \\
\text { DDT: } 4.3-59 \mu \mathrm{g} / \mathrm{g} \\
\text { PCBs: } 5.9-86 \mu \mathrm{g} / \mathrm{g} \\
\text { Chlordanes: } 0.27-5.1 \mu \mathrm{g} / \mathrm{g} \\
\text { HCH: } 0.045-0.43 \mu \mathrm{g} / \mathrm{g}\end{array}$ \\
\hline [40] & 2009 & $\begin{array}{c}\text { Galapagos Islands } \\
\text { (Ecuador) }\end{array}$ & $\begin{array}{l}\text { Galapagos sea lion } \\
\text { (Zalophus wollebaeki) }\end{array}$ & Muscle-Blubber & 21 & $\begin{array}{l}\text { PCBs, } \\
\text { PBDEs, } \\
\text { PCDDs, } \\
\text { PCDFs }\end{array}$ & $\begin{array}{c}\text { PBDEs: Trace } \\
\text { PCDDs \& PCDFs: Not detected } \\
\text { PCBs: } 104 \mu \mathrm{g} / \mathrm{kg}\end{array}$ \\
\hline$[41]$ & 2012 & $\begin{array}{l}\text { Strait of Georgia, } \\
\text { BC }\end{array}$ & $\begin{array}{c}\text { Stellar sea lions } \\
\text { (Eumetopias jubatus) }\end{array}$ & Blubber & 22 & $\begin{array}{l}\text { PBDEs, } \\
\text { PCBs }\end{array}$ & $\begin{array}{l}\text { PBDEs: } 50-3780 \mu \mathrm{g} / \mathrm{kg} \\
\text { PCBs: } 272-14280 \mu \mathrm{g} / \mathrm{kg}\end{array}$ \\
\hline [42] & $\begin{array}{l}1999- \\
2003\end{array}$ & $\begin{array}{l}\text { Hudson Bay, } \\
\text { Canada }\end{array}$ & $\begin{array}{l}\text { Ringed Seal (Pusa } \\
\text { hispida) }\end{array}$ & Blubber & 11 Males & $\begin{array}{l}\text { PBCs, } \\
\text { DDTs, } \\
\text { HCHs, } \\
\text { CBz } \\
\text { Cyclodienes, } \\
\text { PBDEs }\end{array}$ & $\begin{array}{c}\text { PCBS: } 602 \mathrm{ng} / \mathrm{g} \\
\text { DDTs: } 413 \mathrm{ng} / \mathrm{g} \\
\text { HCHs: } 145 \mathrm{ng} / \mathrm{g} \\
\text { CBz: } 78 \mathrm{ng} / \mathrm{g} \\
\text { Cyclodienes: } 157 \mathrm{ng} / \mathrm{g} \\
\text { PBDEs: } 11 \mathrm{ng} / \mathrm{g}\end{array}$ \\
\hline [43] & $\begin{array}{l}2001- \\
2002\end{array}$ & $\begin{array}{l}\text { San Francisco } \\
\text { Bay }\end{array}$ & $\begin{array}{l}\text { Harbor seal (Phoca } \\
\text { vitulina) }\end{array}$ & Blood & 33 & PBDEs & PBDEs: 760 ng/g \\
\hline [44] & $\begin{array}{l}1993- \\
2003\end{array}$ & California & $\begin{array}{l}\text { California sea } \\
\text { lion (Zalophus } \\
\text { californianus) }\end{array}$ & Blubber & 25 Males & PBDEs & PBDEs: 5036 mg/g \\
\hline [43] & $\begin{array}{l}2000- \\
2001\end{array}$ & Bristol Bay, Alaska & $\begin{array}{c}\text { Spotted seal (Phoca } \\
\text { largha) }\end{array}$ & Blood & $\begin{array}{l}5 \text { Females } \\
2 \text { Males }\end{array}$ & PBDEs & $\begin{array}{c}\text { PBDEs: } \\
\text { Females: } 0.280 \mathrm{ng} / \mathrm{g} \\
\text { Males: } 0.456 \mathrm{ng} / \mathrm{g}\end{array}$ \\
\hline$[45]$ & 2003 & $\begin{array}{l}\text { Bering Sea, } \\
\text { Alaska }\end{array}$ & $\begin{array}{c}\text { Bearded seal } \\
\text { (Erignathus barbatus) }\end{array}$ & Blubber & 5 & PBDEs & PBDE: 3.42 ng/g \\
\hline [45] & 2003 & $\begin{array}{l}\text { Bering Sea, } \\
\text { Alaska }\end{array}$ & $\begin{array}{l}\text { Ringed seal (Phoca } \\
\text { hispida) }\end{array}$ & Blubber & 6 & PBDEs & PBDEs: 5.85 ng/g \\
\hline$[45]$ & 2003 & $\begin{array}{l}\text { Bering Sea, } \\
\text { Alaska }\end{array}$ & $\begin{array}{c}\text { Spotted seal (Phoca } \\
\text { largha) }\end{array}$ & Blubber & 3 & PBDEs & PBDEs: $12.4 \mathrm{ng} / \mathrm{g}$ \\
\hline [45] & 2003 & $\begin{array}{l}\text { Bering Sea, } \\
\text { Alaska }\end{array}$ & $\begin{array}{l}\text { Ribbon seal (Phoca } \\
\text { fasciata) }\end{array}$ & Blubber & 6 & PBDEs & PBDEs: $16.5 \mathrm{ng} / \mathrm{g}$ \\
\hline [10] & $\begin{array}{l}1996- \\
1997\end{array}$ & $\begin{array}{l}\text { Queen Charlotte } \\
\text { Strait, Canada }\end{array}$ & $\begin{array}{c}\text { Harbor seal (Phoca } \\
\text { vitulina) }\end{array}$ & Blubber & 60 pups & $\begin{array}{l}\text { PCBs, } \\
\text { PCDDs, } \\
\text { PCDFs }\end{array}$ & $\begin{array}{l}\text { PCBs: } 1,143 \pm 262 \mu \mathrm{g} / \mathrm{kg} \\
\text { PCDDs: } 96 \pm 10 \mathrm{ng} / \mathrm{kg} \\
\text { PCDFs: } 26 \pm 4 \mathrm{ng} / \mathrm{kg}\end{array}$ \\
\hline [10] & $\begin{array}{l}1996- \\
1997\end{array}$ & $\begin{array}{l}\text { Strait of Georgia, } \\
\text { Canada }\end{array}$ & $\begin{array}{c}\text { Harbor seal (Phoca } \\
\text { vitulina) }\end{array}$ & Blubber & 60 pups & $\begin{array}{l}\text { PCBs, } \\
\text { PCDDs, } \\
\text { PCDFs }\end{array}$ & $\begin{array}{l}\text { PCBs: } 2,475 \pm 174 \mu \mathrm{g} / \mathrm{kg} \\
\text { PCDDs: } 279 \pm 32 \mathrm{ng} / \mathrm{kg} \\
\text { PCDFs: } 25 \pm 13 \mathrm{ng} / \mathrm{kg}\end{array}$ \\
\hline [10] & $\begin{array}{l}1996- \\
1997\end{array}$ & Puget Sound, WA & $\begin{array}{c}\text { Harbor seal (Phoca } \\
\text { vitulina) }\end{array}$ & Blubber & 60 pups & $\begin{array}{l}\text { PCBs, } \\
\text { PCDDs, } \\
\text { PCDFs }\end{array}$ & $\begin{array}{l}\text { PCBs: } 18,135 \pm 3,082 \mu \mathrm{g} / \mathrm{kg} \\
\text { PCDDs: } 119 \pm 16 \mathrm{ng} / \mathrm{kg} \\
\text { PCDFs: } 10 \pm 1 \mathrm{ng} / \mathrm{kg}\end{array}$ \\
\hline$[46]$ & $\begin{array}{c}1989- \\
1998\end{array}$ & $\begin{array}{l}\text { San Francisco } \\
\text { Bay, CA }\end{array}$ & $\begin{array}{l}\text { Harbor seal (Phoca } \\
\text { vitulina Richardsi) }\end{array}$ & Blubber & 34 & PBDEs & $\begin{array}{l}\text { All adults: } 1730 \mathrm{ng} / \mathrm{g} \\
\text { Females: } 449 \mathrm{ng} / \mathrm{g} \\
\text { Males: } 2583 \mathrm{ng} / \mathrm{g}\end{array}$ \\
\hline
\end{tabular}


Citation: Green A, Larson S (2016) A Review of Organochlorine Contaminants in Nearshore Marine Mammal Predators. J Environ Anal Toxicol 6: 370. doi:10.4172/2161-0525.1000370

Page 4 of 14

\begin{tabular}{|c|c|c|c|c|c|c|c|}
\hline [47] & $\begin{array}{l}2001 \& \\
2003\end{array}$ & Hornby Island, BC & $\begin{array}{c}\text { Harbor seal (Phoca } \\
\text { vitulina) }\end{array}$ & Blubber & 32 pups & $\begin{array}{l}\text { PCBs, } \\
\text { PBDEs, } \\
\text { PCDEs, } \\
\text { PCNs }\end{array}$ & $\begin{array}{c}2001: \\
\text { PCBs: } 793.3 \pm 147.1 \mu \mathrm{g} / \mathrm{kg} \\
2003: \\
\text { PBDEs: } 582.6 \pm 64.9 \mu \mathrm{g} / \mathrm{kg} \\
\text { PCDEs: } 11.6 \pm 0.9 \mu \mathrm{g} / \mathrm{kg} \\
\text { PCNs: } 1.96 \pm 0.25 \mu \mathrm{g} / \mathrm{kg}\end{array}$ \\
\hline [47] & $\begin{array}{l}2001 \& \\
2003\end{array}$ & Vancouver, BC & $\begin{array}{c}\text { Harbor seal (Phoca } \\
\text { vitulina) }\end{array}$ & Blubber & 32 pups & $\begin{array}{l}\text { PCBs, } \\
\text { PBDEs, } \\
\text { PCDEs, } \\
\text { PCNs }\end{array}$ & $\begin{array}{c}2001: \\
\text { PCBs: } 1106.0 \pm 257.1 \mu \mathrm{g} / \mathrm{kg} \\
2003: \\
\text { PBDEs: } 489.8 \pm 93.9 \mu \mathrm{g} / \mathrm{kg} \\
\text { PCDEs: } 8.9 \pm 1.5 \mu \mathrm{g} / \mathrm{kg} \\
\text { PCNs: } 1.04 \pm 0.13 \mu \mathrm{g} / \mathrm{kg}\end{array}$ \\
\hline [47] & 2003 & Smith Island, WA & $\begin{array}{c}\text { Harbor seal (Phoca } \\
\text { vitulina) }\end{array}$ & Blubber & 32 pups & $\begin{array}{l}\text { PCBs, } \\
\text { PBDEs, } \\
\text { PCDEs, } \\
\text { PCNs }\end{array}$ & $\begin{array}{c}\text { PCBs: } 768.4 \pm 160.6 \mu \mathrm{g} / \mathrm{kg} \\
\text { PBDEs: } 713.9 \pm 228.3 \mu \mathrm{g} / \mathrm{kg} \\
\text { PCDEs: } 6.5 \pm 0.6 \mu \mathrm{g} / \mathrm{kg} \\
\text { PCNs: } 0.84 \pm 0.11 \mu \mathrm{g} / \mathrm{kg}\end{array}$ \\
\hline [47] & 2003 & $\begin{array}{c}\text { Gertrude Island, } \\
\text { WA }\end{array}$ & $\begin{array}{c}\text { Harbor seal (Phoca } \\
\text { vitulina) }\end{array}$ & Blubber & 32 pups & $\begin{array}{l}\text { PCBs, } \\
\text { PBDEs, } \\
\text { PCDEs, } \\
\text { PCNs }\end{array}$ & $\begin{array}{c}\text { PCBs: } 3979.5 \pm 590.3 \mu \mathrm{g} / \mathrm{kg} \\
\text { PBDEs: } 1046.9 \pm 130.2 \mu \mathrm{g} / \mathrm{kg} \\
\text { PCDEs: } 21.07 \pm 2.72 \mu \mathrm{g} / \mathrm{kg} \\
\text { PCNs: } 0.19 \pm 0.05 \mu \mathrm{g} / \mathrm{kg}\end{array}$ \\
\hline [48] & 2009 & Puget Sound, WA & $\begin{array}{c}\text { Harbor seal (Phoca } \\
\text { vitulina) }\end{array}$ & Blubber & 24 pups & $\begin{array}{l}\text { PCBs, } \\
\text { PBDEs }\end{array}$ & $\begin{array}{c}\text { PCBs: } 1.0-9.4 \mu \mathrm{g} / \mathrm{g} \\
\text { PBDEs: } 0.141 .28 \mu \mathrm{g} / \mathrm{g}\end{array}$ \\
\hline [49] & $\begin{array}{l}2009- \\
2010\end{array}$ & Svalbard, Norway & $\begin{array}{c}\text { Harbor seal (Phoca } \\
\text { vitulina) }\end{array}$ & Plasma & 12 & $\begin{array}{l}\text { PCBs, } \\
\text { CHLs, } \\
\text { DDT, } \\
\text { HCH, } \\
\text { HCB, } \\
\text { Mirex }\end{array}$ & $\begin{array}{c}\text { Male: } \\
\text { PCB: } 474 \mathrm{ng} / \mathrm{g} \\
\text { CHL: } 162 \mathrm{ng} / \mathrm{g} \\
\text { DDT: } 118 \mathrm{ng} / \mathrm{g} \\
\text { HCH: } 1.5 \mathrm{ng} / \mathrm{g} \\
\text { HCB: } 2.9 \mathrm{ng} / \mathrm{g} \\
\text { Mirex: } 14 \mathrm{ng} / \mathrm{g} \\
\text { Female: } \\
\text { PCB: } 497 \mathrm{ng} / \mathrm{g} \\
\text { CHL: } 152 \mathrm{ng} / \mathrm{g} \\
\text { DDT: } 222 \mathrm{ng} / \mathrm{g} \\
\text { HCH: } 2.9 \mathrm{ng} / \mathrm{g} \\
\text { HCB: } 4.3 \mathrm{ng} / \mathrm{g} \\
\text { Mirex: } 16 \mathrm{ng} / \mathrm{g} \\
\text { Juvenile: } \\
\text { PCB: } 274 \mathrm{ng} / \mathrm{g} \\
\text { CHL: } 105 \mathrm{ng} / \mathrm{g} \\
\text { DDT: } 128 \mathrm{ng} / \mathrm{g} \\
\text { HCH: } 2.1 \mathrm{ng} / \mathrm{g} \\
\text { HCB: } 3.9 \mathrm{ng} / \mathrm{g} \\
\text { Mirex: } 7.4 \mathrm{ng} / \mathrm{g}\end{array}$ \\
\hline$[6]$ & $\begin{array}{l}2004- \\
2006\end{array}$ & $\begin{array}{c}\text { King George } \\
\text { Island, Antarctica }\end{array}$ & $\begin{array}{l}\text { Weddell seal } \\
\text { (Leptonychotes } \\
\text { weddellii) }\end{array}$ & Blubber & 2 & $\begin{array}{l}\text { PBDEs, } \\
\text { PBCs, } \\
\text { DDTs, } \\
\text { HCBs, } \\
\text { Mirex }\end{array}$ & $\begin{array}{l}\text { PBDEs: } 2.04 \mathrm{ng} / \mathrm{g} \text { I } \\
\text { PCBs: } 300 \mathrm{ng} / \mathrm{g} \\
\text { DDTs: } 131 \mathrm{ng} / \mathrm{g} \\
\text { HCBs: } 5.77 \mathrm{ng} / \mathrm{g} \\
\text { Mirex: } 5.53 \mathrm{ng} / \mathrm{g}\end{array}$ \\
\hline$[6]$ & $\begin{array}{l}2004- \\
2006\end{array}$ & $\begin{array}{c}\text { King George } \\
\text { Island, Antarctica }\end{array}$ & $\begin{array}{l}\text { Antarctic fur seal } \\
\text { (Arctocephalus } \\
\text { gazella) }\end{array}$ & Blubber & 3 & $\begin{array}{l}\text { PBCs, } \\
\text { DDTs, } \\
\text { HCBs, } \\
\text { Mirex }\end{array}$ & $\begin{array}{l}\text { PCBs: } 523 \mathrm{ng} / \mathrm{g} \\
\text { DDTs: } 168 \mathrm{ng} / \mathrm{g} \\
\text { HCBs: } 4.72 \mathrm{ng} / \mathrm{g} \\
\text { Mirex: } 17.0 \mathrm{ng} / \mathrm{g}\end{array}$ \\
\hline$[6]$ & $\begin{array}{l}2004- \\
2006\end{array}$ & $\begin{array}{c}\text { King George } \\
\text { Island, Antarctica }\end{array}$ & $\begin{array}{c}\text { Crabeater } \\
\text { seal (Lobodon } \\
\text { carcinophagus) }\end{array}$ & Blubber & 2 & $\begin{array}{l}\text { PBCs, } \\
\text { DDTs, } \\
\text { HCBs, } \\
\text { Mirex }\end{array}$ & $\begin{array}{l}\text { PCBs: } 154 \mathrm{ng} / \mathrm{g} \\
\text { DDTs: } 14.4 \mathrm{ng} / \mathrm{g} \\
\text { HCBs: } 7.23 \mathrm{ng} / \mathrm{g} \\
\text { Mirex: } 14.4 \mathrm{ng} / \mathrm{g}\end{array}$ \\
\hline$[6]$ & $\begin{array}{l}2004- \\
2006\end{array}$ & $\begin{array}{c}\text { King George } \\
\text { Island, Antarctica }\end{array}$ & $\begin{array}{l}\text { Southern elephant } \\
\text { seal (Mirounga } \\
\text { leonina) }\end{array}$ & Liver & 1 & $\begin{array}{l}\text { PBCs, } \\
\text { DDTs, } \\
\text { HCBs, } \\
\text { Mirex }\end{array}$ & $\begin{array}{l}\text { PCBs: } 73.9 \mathrm{ng} / \mathrm{g} \\
\text { DDTs: } 98.7 \mathrm{ng} / \mathrm{g} \\
\text { HCBs: } 7.48 \mathrm{ng} / \mathrm{g} \\
\text { Mirex: } 16.2 \mathrm{ng} / \mathrm{g}\end{array}$ \\
\hline [50] & $\begin{array}{l}2002- \\
2007\end{array}$ & Svalbard, Norway & $\begin{array}{c}\text { Ringed seal (Phoca } \\
\text { hispida) }\end{array}$ & Liver & 18 & $\begin{array}{c}\text { DDE, } \\
\text { HCB, } \\
\text { Toxaphene }\end{array}$ & $\begin{array}{c}\text { DDE: } 14 \mathrm{ng} / \mathrm{g} \\
\text { HCB: } 0.25 \mathrm{ng} / \mathrm{g} \\
\text { Toxaphene: } 2.8 \mathrm{ng} / \mathrm{g}\end{array}$ \\
\hline [50] & $\begin{array}{l}2002- \\
2007\end{array}$ & Baltic & $\begin{array}{c}\text { Ringed seal (Phoca } \\
\text { hispida) }\end{array}$ & Liver & 31 & $\begin{array}{c}\text { DDE, } \\
\text { HCB, } \\
\text { Toxaphene }\end{array}$ & $\begin{array}{c}\text { DDE: } 113 \mathrm{ng} / \mathrm{g} \\
\text { HCB: } 0.41 \mathrm{ng} / \mathrm{g} \\
\text { Toxaphene: } 5.2 \mathrm{ng} / \mathrm{g}\end{array}$ \\
\hline [51] & $\begin{array}{l}1997- \\
1998\end{array}$ & Baltic & $\begin{array}{c}\text { Gray seal } \\
\text { (Halichoerus grypus) }\end{array}$ & Liver & 12 & $\begin{array}{l}\text { PCBs, } \\
\text { DDT }\end{array}$ & $\begin{array}{l}\text { PCBs: } 25.1 \mu \mathrm{g} / \mathrm{g} \\
\text { DDT: } 7.70 \mu \mathrm{g} / \mathrm{g}\end{array}$ \\
\hline [51] & $\begin{array}{l}1997- \\
1998\end{array}$ & Canada & $\begin{array}{c}\text { Gray seal } \\
\text { (Halichoerus grypus) }\end{array}$ & Liver & 18 & $\begin{array}{l}\text { PCBs, } \\
\text { DDT }\end{array}$ & $\begin{array}{l}\text { PCBs: } 9.59 \mu \mathrm{g} / \mathrm{g} \\
\text { DDT: } 1.82 \mu \mathrm{g} / \mathrm{g}\end{array}$ \\
\hline [51] & $\begin{array}{l}1997- \\
1998\end{array}$ & Baltic & $\begin{array}{c}\text { Ringed seal (Phoca } \\
\text { hispida botnica) }\end{array}$ & Liver & 20 & $\begin{array}{l}\text { PCBs, } \\
\text { DDT }\end{array}$ & $\begin{array}{l}\text { PCBs: } 66.2 \mu \mathrm{g} / \mathrm{g} \\
\text { DDT: } 38.0 \mu \mathrm{g} / \mathrm{g}\end{array}$ \\
\hline
\end{tabular}


Citation: Green A, Larson S (2016) A Review of Organochlorine Contaminants in Nearshore Marine Mammal Predators. J Environ Anal Toxicol 6: 370. doi:10.4172/2161-0525.1000370

Page 5 of 14

\begin{tabular}{|c|c|c|c|c|c|c|c|}
\hline$[51]$ & $\begin{array}{l}1997- \\
1998\end{array}$ & Canada & $\begin{array}{l}\text { Ringed seal (Phoca } \\
\text { hispida botnica) }\end{array}$ & Liver & 26 & $\begin{array}{l}\text { PCBs, } \\
\text { DDT }\end{array}$ & $\begin{array}{l}\text { PCBs: } 0.81 \mu \mathrm{g} / \mathrm{g} \\
\text { DDT: } 0.34 \mu \mathrm{g} / \mathrm{g}\end{array}$ \\
\hline [52] & $\begin{array}{l}2001- \\
2002\end{array}$ & East Greenland & $\begin{array}{l}\text { Ringed seal (Phoca } \\
\text { hispida botnica) }\end{array}$ & Blubber & $\begin{array}{l}6 \text { Females } \\
9 \text { Males }\end{array}$ & $\begin{array}{l}\text { PCBs, } \\
\text { CHLs, } \\
\text { PBDEs }\end{array}$ & $\begin{array}{c}\text { PCBs: } 686 \mathrm{ng} / \mathrm{g} \\
\text { Chlordanes: } 241 \mathrm{ng} / \mathrm{g} \\
\text { PBDEs: } 149 \mathrm{ng} / \mathrm{g}\end{array}$ \\
\hline [53] & $\begin{array}{l}1999- \\
2004\end{array}$ & East Greenland & $\begin{array}{c}\text { Ringed seal (Phoca } \\
\text { hispida) }\end{array}$ & Blubber & 20 & $\begin{array}{l}\text { PCBs, } \\
\text { PBDEs }\end{array}$ & $\begin{array}{l}\text { PCBs: } 596-680 \mathrm{ng} / \mathrm{g} \\
\text { PBDEs: } 31-38 \mathrm{ng} / \mathrm{g}\end{array}$ \\
\hline [23] & & East Greenland & $\begin{array}{c}\text { Ringed seal (Phoca } \\
\text { hispida) }\end{array}$ & Blubber & 5 & $\begin{array}{c}\text { PCBs, } \\
\text { DDTs, } \\
\text { Toxaphene, } \\
\text { CHL, } \\
\text { HCH, } \\
\text { HCB, } \\
\text { PBDEs }\end{array}$ & $\begin{array}{l}\text { PCBs: } 1,370 \mathrm{ng} / \mathrm{g} \\
\text { DDTs: } 1,200 \mathrm{ng} / \mathrm{g} \\
\text { Toxaphene: } 38 \mathrm{ng} / \mathrm{g} \\
\text { CHL: } 400 \mathrm{ng} / \mathrm{g} \\
\text { HCH: } 67 \mathrm{ng} / \mathrm{g} \\
\text { HCB: } 16 \mathrm{ng} / \mathrm{g} \\
\text { PBDEs: } 38 \mathrm{ng} / \mathrm{g}\end{array}$ \\
\hline [18] & $\begin{array}{l}1991- \\
2005\end{array}$ & Northwest Atlantic & $\begin{array}{l}\text { Harbor seal (Phoca } \\
\text { vitulina concolor) }\end{array}$ & Liver & 56 & PBDE & PBDE: $2,670 \mathrm{ng} / \mathrm{g}$ \\
\hline [54] & $\begin{array}{l}1991- \\
2005\end{array}$ & Northwest Atlantic & $\begin{array}{c}\text { Harbor seal (Phoca } \\
\text { vitulina concolor) }\end{array}$ & Blubber & 42 & PBDE & PBDE: $2,403 \mathrm{ng} / \mathrm{g}$ \\
\hline [43] & $\begin{array}{l}2001- \\
2002\end{array}$ & $\begin{array}{c}\text { San Francisco } \\
\text { Bay, CA }\end{array}$ & $\begin{array}{c}\text { Harbor seal (Phoca } \\
\text { vitulina) }\end{array}$ & Blood & 35 & $\begin{array}{l}\text { PCBs, } \\
\text { PBDEs, } \\
\text { DDE }\end{array}$ & $\begin{array}{l}\text { PCBs: } 5,335 \mathrm{ng} / \mathrm{g} \\
\text { PBDEs: } 760 \mathrm{ng} / \mathrm{g} \\
\text { DDE: } 6,806 \mathrm{ng} / \mathrm{g}\end{array}$ \\
\hline & & & & & & & \\
\hline [55] & $\begin{array}{l}1994- \\
2009\end{array}$ & Brazilian Coast & $\begin{array}{c}\text { Franciscana } \\
\text { dolphin (Pontoporia } \\
\text { blainvillei) }\end{array}$ & Liver & 53 & PBDEs, & PBDEs: 7-1797 ng/g \\
\hline$[56]$ & $\begin{array}{l}2003- \\
2004\end{array}$ & $\begin{array}{l}\text { Indian River } \\
\text { Lagoon, FL }\end{array}$ & $\begin{array}{l}\text { Bottlenose dolphin } \\
\text { (Tursiops truncatus) }\end{array}$ & Blubber & 58 & PBDEs & PBDEs: $5,860 \mathrm{ng} / \mathrm{g}$ \\
\hline$[56]$ & $\begin{array}{l}2003- \\
2004\end{array}$ & $\begin{array}{c}\text { Charleston Harbor } \\
\text { estuary, SC }\end{array}$ & $\begin{array}{l}\text { Bottlenose dolphin } \\
\text { (Tursiops truncatus) }\end{array}$ & Blubber & 53 & PBDEs & PBDEs: $1,260 \mathrm{ng} / \mathrm{g}$ \\
\hline$[57]$ & $\begin{array}{l}2000- \\
2002\end{array}$ & Mediterranean & $\begin{array}{l}\text { Striped dolphin } \\
\quad \text { (Stenella } \\
\text { coeruleoalba) }\end{array}$ & Blubber & $\begin{array}{c}6(2000) \\
31(2001) \\
5(2002)\end{array}$ & $\begin{array}{l}\text { DDT, } \\
\text { PCBs }\end{array}$ & $\begin{array}{c}2000: \\
\text { DDT: } 56.22 \mu \mathrm{g} / \mathrm{g} \\
\text { PCB: } 90.4 \mu \mathrm{g} / \mathrm{g} \\
2001: \\
\text { DDT: } 38.11 \mu \mathrm{g} / \mathrm{g} \\
\text { PCB: } 54.42 \mu \mathrm{g} / \mathrm{g} \\
2002: \\
\text { DDT: } 55.09 \mu \mathrm{g} / \mathrm{g} \\
\text { PCB: } 75.90 \mu \mathrm{g} / \mathrm{g}\end{array}$ \\
\hline [58] & $\begin{array}{l}1997- \\
2011\end{array}$ & $\begin{array}{l}\text { Pacific Islands } \\
\text { (Hawaiian, } \\
\text { Mariana, } \\
\text { Micronesia) }\end{array}$ & $\begin{array}{c}\text { Killer whale (Orcinus } \\
\text { orca) }\end{array}$ & Blubber & 1 & $\begin{array}{l}\text { DDT, } \\
\text { PCBs, } \\
\text { CHL, } \\
\text { Toxaphenes, } \\
\text { PBDEs } \\
\text { Mirex, } \\
\text { HCB, } \\
\text { HCHs, } \\
\text { HBCDs }\end{array}$ & $\begin{array}{l}\text { DDT: } 171,000 \mathrm{ng} / \mathrm{g} \\
\text { PCBs: } 93,200 \mathrm{ng} / \mathrm{g} \\
\text { Chlordanes: } 13,600 \mathrm{ng} / \mathrm{g} \\
\text { Toxaphenes: } 6,890 \mathrm{ng} / \mathrm{g} \\
\text { PBDEs: } 938 \mathrm{ng} / \mathrm{g} \\
\text { Mirex: } 6330 \mathrm{ng} / \mathrm{g} \\
\text { HCB: } 580 \mathrm{ng} / \mathrm{g} \\
\text { HCHs: } 187 \mathrm{ng} / \mathrm{g} \\
\text { HBCDs: } 213 \mathrm{ng} / \mathrm{g}\end{array}$ \\
\hline [58] & $\begin{array}{l}1997- \\
2011\end{array}$ & $\begin{array}{l}\text { Pacific Islands } \\
\text { (Hawaiian, } \\
\text { Mariana, } \\
\text { Micronesia) }\end{array}$ & $\begin{array}{c}\text { False killer whale } \\
\text { (Pseudorca } \\
\text { crassidens) }\end{array}$ & Blubber & 1 & $\begin{array}{l}\text { DDT, } \\
\text { PCBs, } \\
\text { CHL, } \\
\text { Toxaphenes, } \\
\text { PBDEs } \\
\text { Mirex, } \\
\text { HCB, } \\
\text { HCHs, } \\
\text { HBCDs }\end{array}$ & $\begin{array}{c}\text { DDT: } 28,200 \mathrm{ng} / \mathrm{g} \\
\text { PCBs: } 26,200 \mathrm{ng} / \mathrm{g} \\
\text { Chlordanes: } 4,900 \mathrm{ng} / \mathrm{g} \\
\text { Toxaphenes: } 2,610 \mathrm{ng} / \mathrm{g} \\
\text { PBDEs: } 1650 \mathrm{ng} / \mathrm{g} \\
\text { Mirex: } 1080 \mathrm{ng} / \mathrm{g} \\
\text { HCB: } 364 \mathrm{ng} / \mathrm{g} \\
\text { HCHs: } 133 \mathrm{ng} / \mathrm{g} \\
\text { HBCDs: } 353 \mathrm{ng} / \mathrm{g}\end{array}$ \\
\hline [58] & $\begin{array}{l}1997- \\
2011\end{array}$ & $\begin{array}{l}\text { Pacific Islands } \\
\text { (Hawaiian, } \\
\text { Mariana, } \\
\text { Micronesia) }\end{array}$ & $\begin{array}{l}\text { Pygmy killer whale } \\
\text { (Feresa attenuata) }\end{array}$ & Blubber & 2 & $\begin{array}{l}\text { DDT, } \\
\text { PCBs, } \\
\text { CHL, } \\
\text { Toxaphenes, } \\
\text { PBDEs } \\
\text { Mirex, } \\
\text { HCB, } \\
\text { HCHs, } \\
\text { HBCDs }\end{array}$ & $\begin{array}{c}\text { DDT: } 24.9-43.8 \mathrm{ng} / \mathrm{g} \\
\text { PCBs: } 2,350-58,200 \mathrm{ng} / \mathrm{g} \\
\text { Chlordanes: } 498-7,590 \mathrm{ng} / \mathrm{g} \\
\text { Toxaphenes: } 518-6,280 \mathrm{ng} / \mathrm{g} \\
\text { PBDEs: } 45.1-521 \mathrm{ng} / \mathrm{g} \\
\text { Mirex: } 136-1820 \mathrm{ng} / \mathrm{g} \\
\text { HCB: } 145-696 \mathrm{ng} / \mathrm{g} \\
\text { HCHs: }<5.86-52.4 \mathrm{ng} / \mathrm{g} \\
\text { HBCDs: } 48.4-248 \mathrm{ng} / \mathrm{g}\end{array}$ \\
\hline
\end{tabular}


Citation: Green A, Larson S (2016) A Review of Organochlorine Contaminants in Nearshore Marine Mammal Predators. J Environ Anal Toxicol 6: 370. doi:10.4172/2161-0525.1000370

Page 6 of 14

\begin{tabular}{|c|c|c|c|c|c|c|c|}
\hline [58] & $\begin{array}{l}1997- \\
2011\end{array}$ & $\begin{array}{l}\text { Pacific Islands } \\
\text { (Hawaiian, } \\
\text { Mariana, } \\
\text { Micronesia) }\end{array}$ & $\begin{array}{c}\text { Melon-headed whale } \\
\text { (Peponocephala } \\
\text { electra) }\end{array}$ & Blubber & 4 & $\begin{array}{l}\text { DDT, } \\
\text { PCBs, } \\
\text { CHL, } \\
\text { Toxaphenes, } \\
\text { PBDEs } \\
\text { Mirex, } \\
\text { HCB, } \\
\text { HCHs, } \\
\text { HBCDs }\end{array}$ & $\begin{array}{c}\text { DDT: } 31,100 \mathrm{ng} / \mathrm{g} \\
\text { PCBs: } 15,600 \mathrm{ng} / \mathrm{g} \\
\text { Chlordanes: } 3,100 \mathrm{ng} / \mathrm{g} \\
\text { Toxaphenes: } 2,510 \mathrm{ng} / \mathrm{g} \\
\text { PBDEs: } 409 \mathrm{ng} / \mathrm{g} \\
\text { Mirex: } 1,190 \mathrm{ng} / \mathrm{g} \\
\text { HCB: } 315 \mathrm{ng} / \mathrm{g} \\
\text { HCHs: } 119 \mathrm{ng} / \mathrm{g} \\
\text { HBCDs: } 108 \mathrm{ng} / \mathrm{g}\end{array}$ \\
\hline [58] & $\begin{array}{l}1997- \\
2011\end{array}$ & $\begin{array}{l}\text { Pacific Islands } \\
\text { (Hawaiian, } \\
\text { Mariana, } \\
\text { Micronesia) }\end{array}$ & $\begin{array}{l}\text { Striped dolphin } \\
\text { (Stenella } \\
\text { coeruleoalba) }\end{array}$ & Blubber & 6 & $\begin{array}{l}\text { DDT, } \\
\text { PCBs, } \\
\text { CHL, } \\
\text { Toxaphenes, } \\
\text { PBDEs } \\
\text { Mirex, } \\
\text { HCB, } \\
\text { HCHs, }\end{array}$ & $\begin{array}{c}\text { DDT: } 20,000 \mathrm{ng} / \mathrm{g} \\
\text { PCBs: } 13,800 \mathrm{ng} / \mathrm{g} \\
\text { Chlordanes: } 2,830 \mathrm{ng} / \mathrm{g} \\
\text { Toxaphenes: } 2,880 \mathrm{ng} / \mathrm{g} \\
\text { PBDEs: } 258 \mathrm{ng} / \mathrm{g} \\
\text { Mirex: } 725 \mathrm{ng} / \mathrm{g} \\
\text { HCB: } 419 \mathrm{ng} / \mathrm{g} \\
\text { HCHs: } 281 \mathrm{ng} / \mathrm{g}\end{array}$ \\
\hline [58] & $\begin{array}{l}1997- \\
2011\end{array}$ & $\begin{array}{l}\text { Pacific Islands } \\
\text { (Hawaiian, } \\
\text { Mariana, } \\
\text { Micronesia) }\end{array}$ & $\begin{array}{l}\text { Rough-toothed } \\
\text { dolphin (Steno } \\
\text { bredanensis) }\end{array}$ & Blubber & 1 & $\begin{array}{l}\text { DDT, } \\
\text { PCBs, } \\
\text { CHL, } \\
\text { Toxaphenes, } \\
\text { PBDEs } \\
\text { Mirex, } \\
\text { HCB, } \\
\text { HCHs, } \\
\text { HBCDs }\end{array}$ & $\begin{array}{c}\text { DDT: } 16,100 \mathrm{ng} / \mathrm{g} \\
\text { PCBs: } 13,800 \mathrm{ng} / \mathrm{g} \\
\text { Chlordanes: } 1,950 \mathrm{ng} / \mathrm{g} \\
\text { Toxaphenes: } 1,230 \mathrm{ng} / \mathrm{g} \\
\text { PBDEs: } 192 \mathrm{ng} / \mathrm{g} \\
\text { Mirex: } 829 \mathrm{ng} / \mathrm{g} \\
\text { HCB: } 98 \mathrm{ng} / \mathrm{g} \\
\text { HCHs: } 33.4 \mathrm{ng} / \mathrm{g} \\
\text { HBCDs: } 80 \mathrm{ng} / \mathrm{g}\end{array}$ \\
\hline [58] & $\begin{array}{l}1997- \\
2011\end{array}$ & $\begin{array}{l}\text { Pacific Islands } \\
\text { (Hawaiian, } \\
\text { Mariana, } \\
\text { Micronesia) }\end{array}$ & $\begin{array}{l}\text { Bottlenose dolphin } \\
\text { (Tursiops truncatus) }\end{array}$ & Blubber & 3 & $\begin{array}{l}\text { DDT, } \\
\text { PCBs, } \\
\text { CHL, } \\
\text { Toxaphenes, } \\
\text { PBDEs } \\
\text { Mirex, } \\
\text { HCB, } \\
\text { HCHs, } \\
\text { HBCDs }\end{array}$ & $\begin{array}{c}\text { DDT: } 15,000 \mathrm{ng} / \mathrm{g} \\
\text { PCBs: } 11,800 \mathrm{ng} / \mathrm{g} \\
\text { Chlordanes: } 1,810 \mathrm{ng} / \mathrm{g} \\
\text { Toxaphenes: } 1,490 \mathrm{ng} / \mathrm{g} \\
\text { PBDEs: } 1,070 \mathrm{ng} / \mathrm{g} \\
\text { Mirex: } 605 \mathrm{ng} / \mathrm{g} \\
\text { HCB: } 378 \mathrm{ng} / \mathrm{g} \\
\text { HCHs: } 136 \mathrm{ng} / \mathrm{g} \\
\text { HBCDs: } 81.7 \mathrm{ng} / \mathrm{g}\end{array}$ \\
\hline [58] & $\begin{array}{l}1997- \\
2011\end{array}$ & $\begin{array}{l}\text { Pacific Islands } \\
\text { (Hawaiian, } \\
\text { Mariana, } \\
\text { Micronesia) }\end{array}$ & $\begin{array}{c}\text { Longman's beaked } \\
\text { whale (Indopacetus } \\
\text { pacificus) }\end{array}$ & Blubber & 1 & $\begin{array}{l}\text { DDT, } \\
\text { PCBs, } \\
\text { CHL, } \\
\text { Toxaphenes, } \\
\text { PBDEs } \\
\text { Mirex, } \\
\text { HCB, } \\
\text { HCHs, } \\
\text { HBCDs }\end{array}$ & $\begin{array}{c}\text { DDT: } 12,000 \mathrm{ng} / \mathrm{g} \\
\text { PCBs: } 7,610 \mathrm{ng} / \mathrm{g} \\
\text { Chlordanes: } 1,690 \mathrm{ng} / \mathrm{g} \\
\text { Toxaphenes: } 1,430 \mathrm{ng} / \mathrm{g} \\
\text { PBDEs: } 118 \mathrm{ng} / \mathrm{g} \\
\text { Mirex: } 139 \mathrm{ng} / \mathrm{g} \\
\text { HCB: } 360 \mathrm{ng} / \mathrm{g} \\
\text { HCHs: } 121 \mathrm{ng} / \mathrm{g} \\
\text { HBCDs: } 62.4 \mathrm{ng} / \mathrm{g}\end{array}$ \\
\hline [58] & $\begin{array}{l}1997- \\
2011\end{array}$ & $\begin{array}{l}\text { Pacific Islands } \\
\text { (Hawaiian, } \\
\text { Mariana, } \\
\text { Micronesia) }\end{array}$ & $\begin{array}{c}\text { Spotted dolphin } \\
\text { (Stenella attenuata) }\end{array}$ & Blubber & 3 & $\begin{array}{l}\text { DDT, } \\
\text { PCBs, } \\
\text { CHL, } \\
\text { Toxaphenes, } \\
\text { PBDEs } \\
\text { Mirex, } \\
\text { HCB, } \\
\text { HCHs, } \\
\text { HBCDs }\end{array}$ & $\begin{array}{c}\text { DDT: } 12,100 \mathrm{ng} / \mathrm{g} \\
\text { PCBs: } 4,610 \mathrm{ng} / \mathrm{g} \\
\text { Chlordanes: } 850 \mathrm{ng} / \mathrm{g} \\
\text { Toxaphenes: } 595 \mathrm{ng} / \mathrm{g} \\
\text { PBDEs: } 78.1 \mathrm{ng} / \mathrm{g} \\
\text { Mirex: } 348 \mathrm{ng} / \mathrm{g} \\
\text { HCB: } 78.9 \mathrm{ng} / \mathrm{g} \\
\text { HCHs: } 21.8 \mathrm{ng} / \mathrm{g} \\
\text { HBCDs: } 21.3 \mathrm{ng} / \mathrm{g}\end{array}$ \\
\hline [58] & $\begin{array}{l}1997- \\
2011\end{array}$ & $\begin{array}{l}\text { Pacific Islands } \\
\text { (Hawaiian, } \\
\text { Mariana, } \\
\text { Micronesia) }\end{array}$ & $\begin{array}{c}\text { Spinner dolphin } \\
\text { (Stenella longirostris) }\end{array}$ & Blubber & 10 & $\begin{array}{l}\text { DDT, } \\
\text { PCBs, } \\
\text { CHL, } \\
\text { Toxaphenes, } \\
\text { PBDEs } \\
\text { Mirex, } \\
\text { HCB, } \\
\text { HCHs, } \\
\text { HBCDs }\end{array}$ & $\begin{array}{c}\text { DDT: } 2,530 \mathrm{ng} / \mathrm{g} \\
\text { PCBs: } 2,090 \mathrm{ng} / \mathrm{g} \\
\text { Chlordanes: } 533 \mathrm{ng} / \mathrm{g} \\
\text { Toxaphenes: } 611 \mathrm{ng} / \mathrm{g} \\
\text { PBDEs: } 559 \mathrm{ng} / \mathrm{g} \\
\text { Mirex: } 107 \mathrm{ng} / \mathrm{g} \\
\text { HCB: } 134 \mathrm{ng} / \mathrm{g} \\
\text { HCHs: } 29 \mathrm{ng} / \mathrm{g} \\
\text { HBCDs: } 20 \mathrm{ng} / \mathrm{g}\end{array}$ \\
\hline [58] & $\begin{array}{l}1997- \\
2011\end{array}$ & $\begin{array}{l}\text { Pacific Islands } \\
\text { (Hawaiian, } \\
\text { Mariana, } \\
\text { Micronesia) }\end{array}$ & $\begin{array}{l}\text { Dwarf sperm whale } \\
\text { (Kogia sima) }\end{array}$ & Blubber & 1 & $\begin{array}{l}\text { DDT, } \\
\text { PCBs, } \\
\text { CHL, } \\
\text { Toxaphenes, } \\
\text { PBDEs } \\
\text { Mirex, } \\
\text { HCB, } \\
\text { HCHs, } \\
\text { HBCDs }\end{array}$ & $\begin{array}{c}\text { DDT: } 3,030 \mathrm{ng} / \mathrm{g} \\
\text { PCBs: } 1,900 \mathrm{ng} / \mathrm{g} \\
\text { Chlordanes: } 313 \mathrm{ng} / \mathrm{g} \\
\text { Toxaphenes: } 113 \mathrm{ng} / \mathrm{g} \\
\text { PBDEs: } 25.5 \mathrm{ng} / \mathrm{g} \\
\text { Mirex: } 106 \mathrm{ng} / \mathrm{g} \\
\text { HCB: } 79.8 \mathrm{ng} / \mathrm{g} \\
\text { HCHs: }<3.24 \mathrm{ng} / \mathrm{g} \\
\text { HBCDs: } 17.2 \mathrm{ng} / \mathrm{g}\end{array}$ \\
\hline
\end{tabular}


Citation: Green A, Larson S (2016) A Review of Organochlorine Contaminants in Nearshore Marine Mammal Predators. J Environ Anal Toxicol 6: 370. doi:10.4172/2161-0525.1000370

Page 7 of 14

\begin{tabular}{|c|c|c|c|c|c|c|c|}
\hline$[58]$ & $\begin{array}{l}1997- \\
2011\end{array}$ & $\begin{array}{l}\text { Pacific Islands } \\
\text { (Hawaiian, } \\
\text { Mariana, } \\
\text { Micronesia) }\end{array}$ & $\begin{array}{l}\text { Blainville's beaked } \\
\text { whale (Mesoplodon } \\
\text { densirostris) }\end{array}$ & Blubber & 1 & $\begin{array}{l}\text { DDT, } \\
\text { PCBs, } \\
\text { CHL, } \\
\text { Toxaphenes, } \\
\text { PBDEs } \\
\text { Mirex, } \\
\text { HCB, } \\
\text { HCHs, } \\
\text { HBCDs }\end{array}$ & $\begin{array}{c}\text { DDT: } 2,480 \mathrm{ng} / \mathrm{g} \\
\text { PCBs: } 1,450 \mathrm{ng} / \mathrm{g} \\
\text { Chlordanes: } 309 \mathrm{ng} / \mathrm{g} \\
\text { Toxaphenes: } 169 \mathrm{ng} / \mathrm{g} \\
\text { PBDEs: } 30.4 \mathrm{ng} / \mathrm{g} \\
\text { Mirex: } 46.2 \mathrm{ng} / \mathrm{g} \\
\text { HCB: } 53.7 \mathrm{ng} / \mathrm{g} \\
\text { HCHs: } 8.07 \mathrm{ng} / \mathrm{g} \\
\text { HBCDs: } 21.6 \mathrm{ng} / \mathrm{g}\end{array}$ \\
\hline [59] & Not given & Brazil & $\begin{array}{c}\text { Guiana dolphin } \\
\text { (Sotalia guianensis) }\end{array}$ & Blubber & $\begin{array}{l}7 \text { Males } \\
4 \text { Females }\end{array}$ & PCBs & $\begin{array}{l}\text { Male: } 100,290 \mathrm{pg} / \mathrm{g} \\
\text { Female: } 107,865 \mathrm{pg} / \mathrm{g}\end{array}$ \\
\hline [59] & Not given & Brazil & $\begin{array}{l}\text { Rough toothed } \\
\text { dolphin (Steno } \\
\text { bredanensis) }\end{array}$ & Blubber & 1 Male & PCBs & $74,705 \mathrm{pg} / \mathrm{g}$ \\
\hline [59] & Not given & Brazil & $\begin{array}{c}\text { False killer whale } \\
\text { (Pseudorca } \\
\text { crassidens) }\end{array}$ & Blubber & 1 Female & PCBs & $122,004 \mathrm{pg} / \mathrm{g}$ \\
\hline$[60]$ & 2002 & $\begin{array}{l}\text { Ligurian and } \\
\text { Ionian Sea }\end{array}$ & $\begin{array}{l}\text { Striped dolphin } \\
\text { (Stenella } \\
\text { coeruleoalba) }\end{array}$ & Skin Biopsy & 18 & $\begin{array}{l}\text { PCBs, } \\
\text { DDT }\end{array}$ & $\begin{array}{l}\text { PCBs: }<40 \mathrm{mg} / \mathrm{kg} \text { dry weight } \\
\text { DDTs: }<30 \mathrm{mg} / \mathrm{kg} \text { dry weight }\end{array}$ \\
\hline$[60]$ & 2002 & $\begin{array}{l}\text { Ligurian and } \\
\text { Ionian Sea }\end{array}$ & $\begin{array}{l}\text { Bottlenose dolphin } \\
\text { (Tursiops truncatus), }\end{array}$ & Skin Biopsy & 1 & $\begin{array}{l}\text { PCBs, } \\
\text { DDT }\end{array}$ & $\begin{array}{l}\text { PCBs: }<20 \mathrm{mg} / \mathrm{kg} \text { dry weight } \\
\text { DDT: }<10 \mathrm{mg} / \mathrm{kg} \text { dry weight }\end{array}$ \\
\hline$[60]$ & 2002 & $\begin{array}{l}\text { Ligurian and } \\
\text { Ionian Sea }\end{array}$ & $\begin{array}{l}\text { Common dolphin } \\
\text { (Delphinus delphis), }\end{array}$ & Skin Biopsy & 3 & $\begin{array}{l}\text { PCBs, } \\
\text { DDT }\end{array}$ & $\begin{array}{l}\text { PCBs: }<20 \mathrm{mg} / \mathrm{kg} \text { dry weight } \\
\text { DDT: }<20 \mathrm{mg} / \mathrm{kg} \text { dry weight }\end{array}$ \\
\hline$[60]$ & 2002 & $\begin{array}{l}\text { Ligurian and } \\
\text { Ionian Sea }\end{array}$ & $\begin{array}{l}\text { Fin whale } \\
\text { (Balenoptera } \\
\text { physalus) }\end{array}$ & Skin Biopsy & 14 & $\begin{array}{l}\text { PCBs, } \\
\text { DDT }\end{array}$ & $\begin{array}{l}\text { PCBs: }<10 \mathrm{mg} / \mathrm{kg} \text { dry weight } \\
\text { DDT: }<10 \mathrm{mg} / \mathrm{kg} \text { dry weight }\end{array}$ \\
\hline$[5]$ & $\begin{array}{l}1994- \\
1999\end{array}$ & $\begin{array}{l}\text { Kenai Fjords/ } \\
\text { Prince William } \\
\text { Sound }\end{array}$ & $\begin{array}{c}\text { Killer whale (Orcinus } \\
\text { orca) }\end{array}$ & Blubber & 77 & $\begin{array}{l}\text { PCBs, } \\
\text { DDT }\end{array}$ & $\begin{array}{c}\text { PCBs: } \\
\text { Resident: } 14,000 \mathrm{ng} / \mathrm{g} \\
\text { Transient: } 230,000 \mathrm{ng} / \mathrm{g} \\
\text { DDT: } \\
\text { Resident: } 13,000 \mathrm{ng} / \mathrm{g} \\
\text { Transient: } 320,000 \mathrm{ng} / \mathrm{g}\end{array}$ \\
\hline [24] & 2002 & Norway & $\begin{array}{c}\text { Killer whale (Orcinus } \\
\text { orca) }\end{array}$ & Blubber & 9 & $\begin{array}{l}\text { PCBs, } \\
\text { PBDEs, } \\
\text { DDE, } \\
\text { CHL, } \\
\text { Toxaphene }\end{array}$ & $\begin{array}{c}\text { PCBs: } 26,940 \mathrm{ng} / \mathrm{g} \\
\text { PBDEs: } 475 \mathrm{ng} / \mathrm{g} \\
\text { DDE: } 11,652 \mathrm{ng} / \mathrm{g} \\
\text { Chlordanes: } 6,565 \mathrm{ng} / \mathrm{g} \\
\text { Toxaphene: } 8,206 \mathrm{ng} / \mathrm{g}\end{array}$ \\
\hline [61] & 2006 & $\begin{array}{l}\text { Georgia Basin, } \\
\text { Canada }\end{array}$ & $\begin{array}{c}\text { Southern resident } \\
\text { killer whale (Orcinus } \\
\text { orca) }\end{array}$ & Blubber & $\begin{array}{l}1 \text { Female } \\
3 \text { Males } \\
\text { (J-Pod) }\end{array}$ & $\begin{array}{l}\text { PCBs, } \\
\text { PBDEs, } \\
\text { DDT, } \\
\text { CHL, } \\
\text { HCHs, } \\
\text { HCB }\end{array}$ & $\begin{array}{c}\text { PCBs: } \\
\text { Female: } 45,000 \mathrm{ng} / \mathrm{g} \\
\text { Male: } 34,000-180,000 \mathrm{ng} / \mathrm{g} \\
\text { PBDEs: } \\
\text { Female: } 7,500 \mathrm{ng} / \mathrm{g} \\
\text { Male: } 6,300-15,000 \mathrm{ng} / \mathrm{g} \\
\text { DDT: } \\
\text { Female: } 26,000 \mathrm{ng} / \mathrm{g} \\
\text { Male: } 24,000-160,000 \mathrm{ng} / \mathrm{g} \\
\text { CHL: } \\
\text { Female: } 4,300 \mathrm{ng} / \mathrm{g} \\
\text { Male: } 5,100-14,000 \mathrm{ng} / \mathrm{g} \\
\text { HCHs: } \\
\text { Female: } 310 \mathrm{ng} / \mathrm{g} \\
\text { Male: } 580-1,300 \mathrm{ng} / \mathrm{g} \\
\text { HCB: } \\
\text { Female: } 160 \mathrm{ng} / \mathrm{g} \\
\text { Male: } 570-1,600 \mathrm{ng} / \mathrm{g}\end{array}$ \\
\hline [61] & $\begin{array}{l}2004- \\
2006\end{array}$ & $\begin{array}{l}\text { Puget Sound, } \\
\text { Washington and } \\
\text { Georgia Basin, } \\
\text { Canada }\end{array}$ & $\begin{array}{c}\text { Southern resident } \\
\text { killer whale (Orcinus } \\
\text { orca) }\end{array}$ & Blubber & $\begin{array}{l}5 \text { Males } \\
\text { (L-Pod) }\end{array}$ & $\begin{array}{l}\text { PCBs, } \\
\text { PBDEs, } \\
\text { DDT, } \\
\text { CHL, } \\
\text { HCHs, } \\
\text { HCB }\end{array}$ & $\begin{array}{c}\text { PCBs: } 22,000-56,000 \mathrm{ng} / \mathrm{g} \\
\text { PBDEs: } 2,500-3,300 \mathrm{ng} / \mathrm{g} \\
\text { DDT: } 38,000-110,000 \mathrm{ng} / \mathrm{g} \\
\text { CHL: } 7,400-12,000 \mathrm{ng} / \mathrm{g} \\
\text { HCHs: } 560-920 \mathrm{ng} / \mathrm{g} \\
\text { HCB: } 520-730 \mathrm{ng} / \mathrm{g}\end{array}$ \\
\hline [19] & 2007 & Puget Sound, WA & $\begin{array}{c}\text { Southern resident } \\
\text { killer whale (Orcinus } \\
\text { orca) }\end{array}$ & Blubber & 2 (J-Pod) & $\begin{array}{l}\text { PCBs, } \\
\text { DDT, } \\
\text { PBDEs, } \\
\text { CHL, } \\
\mathrm{HCH}\end{array}$ & $\begin{array}{l}\text { PCBs: } 4,600-41,000 \mathrm{ng} / \mathrm{g} \\
\text { DDT: } 1,500-24,000 \mathrm{ng} / \mathrm{g} \\
\text { PBDEs: } 880-14,000 \mathrm{ng} / \mathrm{g} \\
\text { CHL: } 290-5,100 \mathrm{ng} / \mathrm{g} \\
\text { HCH: } 62-1000 \mathrm{ng} / \mathrm{g}\end{array}$ \\
\hline [19] & 2007 & Puget Sound, WA & $\begin{array}{c}\text { Southern resident } \\
\text { killer whale (Orcinus } \\
\text { orca) }\end{array}$ & Blubber & 5 (K-pod) & $\begin{array}{l}\text { PCBs, } \\
\text { DDT, } \\
\text { PBDEs, } \\
\text { CHL, } \\
\text { HCH }\end{array}$ & $\begin{array}{c}\text { PCBs: } 8,900-120,000 \mathrm{ng} / \mathrm{g} \\
\text { DDT: } 11,000-95,000 \mathrm{ng} / \mathrm{g} \\
\text { PBDEs: } 1,200-15,000 \mathrm{ng} / \mathrm{g} \\
\text { CHL: } 1.400-16,000 \mathrm{ng} / \mathrm{g} \\
\text { HCH: } 300-1,700 \mathrm{ng} / \mathrm{g}\end{array}$ \\
\hline
\end{tabular}


Citation: Green A, Larson S (2016) A Review of Organochlorine Contaminants in Nearshore Marine Mammal Predators. J Environ Anal Toxicol 6: 370. doi:10.4172/2161-0525.1000370

Page 8 of 14

\begin{tabular}{|c|c|c|c|c|c|c|c|}
\hline [19] & 2007 & Puget Sound, WA & $\begin{array}{c}\text { Southern resident } \\
\text { killer whale (Ornius } \\
\text { orca) }\end{array}$ & Blubber & 5 (L-pod) & $\begin{array}{l}\text { PCBs, } \\
\text { DDT, } \\
\text { PBDEs, } \\
\text { CHL, } \\
\text { HCH }\end{array}$ & $\begin{array}{c}\text { PCBs: } 5,600-55,000 \mathrm{ng} / \mathrm{g} \\
\text { DDT: } 4,300-99,000 \mathrm{ng} / \mathrm{g} \\
\text { PBDEs: } 680-4,400 \mathrm{ng} / \mathrm{g} \\
\text { CHL: } 730-9,500 \mathrm{ng} / \mathrm{g} \\
\text { HCH: } 150-750 \mathrm{ng} / \mathrm{g}\end{array}$ \\
\hline [42] & $\begin{array}{l}1999- \\
2003\end{array}$ & $\begin{array}{l}\text { Hudson Bay, } \\
\text { Canada }\end{array}$ & $\begin{array}{c}\text { Beluga whale } \\
\text { (Delphin apterus } \\
\text { leucas) }\end{array}$ & Blubber & $\begin{array}{l}9 \text { Calves, } \\
14 \text { Females } \\
21 \text { males }\end{array}$ & $\begin{array}{l}\text { PBCs, } \\
\text { DDTs, } \\
\text { HCHs, } \\
\text { CBz } \\
\text { Cyclodienes, } \\
\text { PBDEs }\end{array}$ & $\begin{array}{c}\text { PCBs: } \\
\text { Calves: } 670 \mathrm{ng} / \mathrm{g} \\
\text { Females: } 661 \mathrm{ng} / \mathrm{g} \\
\text { Males: } 3,690 \mathrm{ng} / \mathrm{g} \\
\text { DDTs: } \\
\text { Calves: } 1030 \mathrm{ng} / \mathrm{g} \\
\text { Females: } 520 \mathrm{ng} / \mathrm{g} \\
\text { Males: } 2,521 \mathrm{ng} / \mathrm{g} \\
\text { HCHs: } \\
\text { Calves: } 307 \mathrm{ng} / \mathrm{g} \\
\text { Females: } 95 \mathrm{ng} / \mathrm{g} \\
\text { Males: } 119 \mathrm{ng} / \mathrm{g} \\
\text { CBz: } \\
\text { Calves: } 487 \mathrm{ng} / \mathrm{g} \\
\text { Females: } 112 \mathrm{ng} / \mathrm{g} \\
\text { Males: } 377 \mathrm{ng} / \mathrm{g} \\
\text { Cyclodienes: } \\
\text { Calves: } 510 \mathrm{ng} / \mathrm{g} \\
\text { Females: } 497 \mathrm{ng} / \mathrm{g} \\
\text { Males: } 473 \mathrm{ng} / \mathrm{g} \\
\text { PBDEs: } \\
\text { Calves: } 27 \mathrm{ng} / \mathrm{g} \\
\text { Females: } 16 \mathrm{ng} / \mathrm{g} \\
\text { Males: } 34 \mathrm{ng} / \mathrm{g}\end{array}$ \\
\hline [62] & $\begin{array}{l}1995- \\
1997\end{array}$ & Svalbard, Norway & $\begin{array}{c}\text { Beluga whale } \\
\text { (Delphin apterus } \\
\text { leucas) }\end{array}$ & Blubber & 10 Males & $\begin{array}{l}\text { HCB, } \\
\text { HCH, } \\
\text { Dieldrin, } \\
\text { DDT, } \\
\text { PCB }\end{array}$ & $\begin{array}{l}\text { HCB: } 430 \mathrm{ng} / \mathrm{g} \\
\text { Dieldrin: } 1005 \mathrm{ng} / \mathrm{g} \\
\text { HCH: } 111 \mathrm{ng} / \mathrm{g} \\
\text { DDT: } 5083 \mathrm{ng} / \mathrm{g} \\
\text { PCBs: } 4680 \mathrm{ng} / \mathrm{g}\end{array}$ \\
\hline [63] & $\begin{array}{l}2002- \\
2003\end{array}$ & $\begin{array}{l}\text { St. Lawrence } \\
\text { River Estuary, } \\
\text { Canada }\end{array}$ & $\begin{array}{c}\text { Beluga whale } \\
\text { (Delphin apterus } \\
\text { leucas) }\end{array}$ & Liver & 6 & $\begin{array}{l}\text { PBDEs, } \\
\text { PCBs, } \\
\text { DDT, } \\
\text { HCH, } \\
\text { CHL, } \\
\text { Mirex, } \\
\text { HCBs, } \\
\text { Dieldrin }\end{array}$ & $\begin{array}{c}\text { PBDEs: } 2,210 \mathrm{ng} / \mathrm{g} \\
\text { PCBs: } 31,937 \mathrm{ng} / \mathrm{g} \\
\text { DDT: } 4,536 \mathrm{ng} / \mathrm{g} \\
\text { HCH: } 74 \mathrm{ng} / \mathrm{g} \\
\text { Chlordanes: } 2,355 \mathrm{ng} / \mathrm{g} \\
\text { Mirex: } 47 \mathrm{ng} / \mathrm{g} \\
\text { HCBs: } 121 \mathrm{ng} / \mathrm{g} \\
\text { Dieldrin: } 757 \mathrm{ng} / \mathrm{g}\end{array}$ \\
\hline [63] & $\begin{array}{l}2002- \\
2003\end{array}$ & $\begin{array}{l}\text { Western Hudson } \\
\text { Bay, Canada }\end{array}$ & $\begin{array}{c}\text { Beluga whale } \\
\text { (Delphin apterus } \\
\text { leucas) }\end{array}$ & Liver & 11 & $\begin{array}{l}\text { PBDEs, } \\
\text { PCBs, } \\
\text { DDT, } \\
\text { HCH, } \\
\text { CHL, } \\
\text { Mirex, } \\
\text { HCBs, } \\
\text { Dieldrin }\end{array}$ & $\begin{array}{c}\text { PBDEs: } 53 \mathrm{ng} / \mathrm{g} \\
\text { PCBs: } 1,1737 \mathrm{ng} / \mathrm{g} \\
\text { DDT: } 284 \mathrm{ng} / \mathrm{g} \\
\text { HCH: } 45 \mathrm{ng} / \mathrm{g} \\
\text { Chlordanes: } 808 \mathrm{ng} / \mathrm{g} \\
\text { Mirex: } 0.59 \mathrm{ng} / \mathrm{g} \\
\text { HCBs: } 144 \mathrm{ng} / \mathrm{g} \\
\text { Dieldrin: } 275 \mathrm{ng} / \mathrm{g}\end{array}$ \\
\hline [64] & $\begin{array}{l}2001- \\
2004\end{array}$ & Gulf of Mexico & $\begin{array}{l}\text { Bottlenose dolphin } \\
\text { (Tursiops truncatus) }\end{array}$ & Blubber & 14 & PBDEs & PBDEs: 817 ng/g \\
\hline [64] & $\begin{array}{l}2001- \\
2004\end{array}$ & Atlantic Coast & $\begin{array}{l}\text { Bottlenose dolphin } \\
\text { (Tursiops truncatus) }\end{array}$ & Blubber & 6 & PBDEs & PBDEs: 1130 ng/g \\
\hline$[65]$ & $\begin{array}{l}2002- \\
2004\end{array}$ & Atlantic Coast (FL) & $\begin{array}{l}\text { Bottlenose dolphin } \\
\text { (Tursiops truncatus) }\end{array}$ & Blubber & 6 Females & $\begin{array}{l}\text { PCBs, } \\
\text { DDT, } \\
\text { CHL, } \\
\text { PBDEs }\end{array}$ & $\begin{array}{l}\text { PCBs: } 891 \mathrm{ng} / \mathrm{g} \\
\text { DDT: } 97 \mathrm{ng} / \mathrm{g} \\
\text { CHL: } 39 \mathrm{ng} / \mathrm{g} \\
\text { PBDEs: } 21 \mathrm{ng} / \mathrm{g}\end{array}$ \\
\hline [65] & $\begin{array}{l}2002- \\
2004\end{array}$ & Atlantic Coast (FL) & $\begin{array}{l}\text { Bottlenose dolphin } \\
\text { (Tursiops truncatus) }\end{array}$ & Blubber & $\begin{array}{l}31 \text { Males and } \\
\text { Juveniles }\end{array}$ & $\begin{array}{l}\text { PCBs, } \\
\text { DDT, } \\
\text { CHL, } \\
\text { PBDEs }\end{array}$ & $\begin{array}{l}\text { PCBs: } 19,900 \mathrm{ng} / \mathrm{g} \\
\text { DDT: } 2,980 \mathrm{ng} / \mathrm{g} \\
\text { CHL: } 1,070 \mathrm{ng} / \mathrm{g} \\
\text { PBDEs: } 394 \mathrm{ng} / \mathrm{g}\end{array}$ \\
\hline$[56]$ & $\begin{array}{l}2003- \\
2004\end{array}$ & Atlantic Coast (FL) & $\begin{array}{l}\text { Bottlenose dolphin } \\
\text { (Tursiops truncatus) }\end{array}$ & Blubber & $\begin{array}{l}16 \text { Females } \\
25 \text { Males } \\
11 \text { Juveniles }\end{array}$ & PBDEs & $\begin{array}{c}\text { PBDEs: } \\
\text { Females: } 718 \mathrm{ng} / \mathrm{g} \\
\text { Males: } 1690 \mathrm{ng} / \mathrm{g} \\
\text { Juveniles: } 979 \mathrm{ng} / \mathrm{g}\end{array}$ \\
\hline$[56]$ & $\begin{array}{l}2003- \\
2004\end{array}$ & $\begin{array}{l}\text { Atlantic Coast } \\
\text { (SC) }\end{array}$ & $\begin{array}{l}\text { Bottlenose dolphin } \\
\text { (Tursiops truncatus) }\end{array}$ & Blubber & $\begin{array}{c}9 \text { Females } \\
31 \text { Males } \\
13 \text { Juveniles }\end{array}$ & PBDEs & $\begin{array}{c}\text { PBDEs: } \\
\text { Females: } 1153 \mathrm{ng} / \mathrm{g} \\
\text { Males: } 6830 \mathrm{ng} / \mathrm{g} \\
\text { Juveniles: } 7055 \mathrm{ng} / \mathrm{g}\end{array}$ \\
\hline
\end{tabular}


Citation: Green A, Larson S (2016) A Review of Organochlorine Contaminants in Nearshore Marine Mammal Predators. J Environ Anal Toxicol 6: 370. doi:10.4172/2161-0525.1000370

Page 9 of 14

\begin{tabular}{|c|c|c|c|c|c|c|c|}
\hline [64] & $\begin{array}{l}1991- \\
1996\end{array}$ & $\begin{array}{c}\text { Florida, western } \\
\text { coast }\end{array}$ & $\begin{array}{l}\text { Bottlenose dolphin } \\
\text { (Tursiops truncatus) }\end{array}$ & Blubber & $\begin{array}{l}11 \text { Males } \\
9 \text { Females }\end{array}$ & $\begin{array}{l}\text { PCBs, } \\
\text { PBDEs }\end{array}$ & $\begin{array}{l}\text { PCBs: } 36,200 \mathrm{ng} / \mathrm{g} \\
\text { PBDEs: } 363 \mathrm{ng} / \mathrm{g}\end{array}$ \\
\hline [64] & $\begin{array}{l}2000- \\
2001\end{array}$ & $\begin{array}{c}\text { Florida, western } \\
\text { coast }\end{array}$ & $\begin{array}{l}\text { Bottlenose dolphin } \\
\text { (Tursiops truncatus) }\end{array}$ & Blubber & 14 & $\begin{array}{l}\text { PCBs, } \\
\text { PBDEs }\end{array}$ & $\begin{array}{l}\text { PCBs: } 240,000 \mathrm{ng} / \mathrm{g} \\
\text { PBDEs: } 1,270 \mathrm{ng} / \mathrm{g}\end{array}$ \\
\hline [64] & $\begin{array}{l}2001- \\
2004\end{array}$ & $\begin{array}{l}\text { Florida, eastern } \\
\text { coast }\end{array}$ & $\begin{array}{l}\text { Bottlenose dolphin } \\
\text { (Tursiops truncatus) }\end{array}$ & Blubber & 6 & $\begin{array}{l}\text { PCBs, } \\
\text { PBDEs }\end{array}$ & $\begin{array}{l}\text { PCBs: } 96,300 \mathrm{ng} / \mathrm{g} \\
\text { PBDEs: } 1,130 \mathrm{ng} / \mathrm{g}\end{array}$ \\
\hline [64] & $\begin{array}{l}2001- \\
2004\end{array}$ & $\begin{array}{c}\text { Florida, western } \\
\text { coast }\end{array}$ & $\begin{array}{l}\text { Striped dolphin } \\
\text { (Stenella } \\
\text { coeruleoalba) }\end{array}$ & Blubber & 1 & $\begin{array}{l}\text { PCBs, } \\
\text { PBDEs }\end{array}$ & $\begin{array}{l}\text { PCBs: } 51,700 \mathrm{ng} / \mathrm{g} \\
\text { PBDEs: } 660 \mathrm{ng} / \mathrm{g}\end{array}$ \\
\hline [66] & 2006 & Korea & $\begin{array}{c}\text { Long-beaked } \\
\text { common dolphin } \\
\text { (Delphinus capensis) }\end{array}$ & Blubber & 22 & $\begin{array}{l}\text { PCBs, } \\
\text { DDTs, } \\
\text { CHL, } \\
\text { HCH, } \\
\text { HCB, } \\
\text { PBDEs }\end{array}$ & $\begin{array}{c}\text { PCBs: } \\
\text { Male: } 15,000 \mathrm{ng} / \mathrm{g} \\
\text { Female: } 15,000 \mathrm{ng} / \mathrm{g} \\
\text { DDTs: } \\
\text { Male: } 14,000 \mathrm{ng} / \mathrm{g} \\
\text { Female: } 13,000 \mathrm{ng} / \mathrm{g} \\
\text { CHL: } \\
\text { Male: } 1,100 \mathrm{ng} / \mathrm{g} \\
\text { Female: } 1,100 \mathrm{ng} / \mathrm{g} \\
\mathrm{HCH}: \\
\text { Male: } 320 \mathrm{ng} / \mathrm{g} \\
\text { Female: } 360 \mathrm{ng} / \mathrm{g} \\
\text { HCB: } \\
\text { Male: } 110 \mathrm{ng} / \mathrm{g} \\
\text { Female: } 100 \mathrm{ng} / \mathrm{g} \\
\text { PBDEs: } \\
\text { Male: } 1,700 \mathrm{ng} / \mathrm{g} \\
\text { Female: } 1,600 \mathrm{ng} / \mathrm{g}\end{array}$ \\
\hline [67] & $\begin{array}{l}2001- \\
2003\end{array}$ & $\begin{array}{c}\text { Gulf of Alaska, } \\
\text { Aleutian Island } \\
\text { and Prince William } \\
\text { Sound }\end{array}$ & $\begin{array}{c}\text { North Pacific killer } \\
\text { whale (Orcinus orca) }\end{array}$ & Blubber & 14 Residents & $\begin{array}{l}\text { PCBs, } \\
\text { DDT, } \\
\text { CHL, } \\
\text { HCHs }\end{array}$ & $\begin{array}{c}\text { PCBS: } 15,000 \mathrm{ng} / \mathrm{g} \\
\text { DDT: } 25,000 \mathrm{ng} / \mathrm{g} \\
\text { Chlordanes: } 5,700 \mathrm{ng} / \mathrm{g} \\
\text { HCHs: } 470 \mathrm{ng} / \mathrm{g}\end{array}$ \\
\hline$[67]$ & $\begin{array}{l}2001- \\
2003\end{array}$ & $\begin{array}{c}\text { Gulf of Alaska, } \\
\text { Aleutian Island } \\
\text { and Prince William } \\
\text { Sound }\end{array}$ & $\begin{array}{c}\text { North Pacific killer } \\
\text { whale (Orcinus orca) }\end{array}$ & Blubber & 2 Offshores & $\begin{array}{l}\text { PCBs, } \\
\text { DDT, } \\
\text { CHL, } \\
\text { HCHs }\end{array}$ & $\begin{array}{c}\text { PCBS: } 66,000 \mathrm{ng} / \mathrm{g} \\
\text { DDT: } 170,000 \mathrm{ng} / \mathrm{g} \\
\text { Chlordanes: } 6,600 \mathrm{ng} / \mathrm{g} \\
\text { HCHs: } 120 \mathrm{ng} / \mathrm{g}\end{array}$ \\
\hline [67] & $\begin{array}{l}2001- \\
2003\end{array}$ & $\begin{array}{c}\text { Gulf of Alaska, } \\
\text { Aleutian Island } \\
\text { and Prince William } \\
\text { Sound }\end{array}$ & $\begin{array}{c}\text { North Pacific killer } \\
\text { whale (Orcinus orca) }\end{array}$ & Blubber & 5 Transients & $\begin{array}{l}\text { PCBs, } \\
\text { DDT, } \\
\text { CHL, } \\
\text { HCHs }\end{array}$ & $\begin{array}{c}\text { PCBS: } 150,000 \mathrm{ng} / \mathrm{g} \\
\text { DDT: } 270,000 \mathrm{ng} / \mathrm{g} \\
\text { Chlordanes: } 72,000 \mathrm{ng} / \mathrm{g} \\
\text { HCHs: } 11,500 \mathrm{ng} / \mathrm{g}\end{array}$ \\
\hline [68] & 2003 & Korean coast & $\begin{array}{c}\text { Finless porpoise } \\
\text { (Neophocaena } \\
\text { phocaenoides) }\end{array}$ & Blubber & $\begin{array}{c}7 \text { Males } \\
10 \text { Females }\end{array}$ & $\begin{array}{l}\text { PCBs, } \\
\text { DDTs, } \\
\text { HCHs, } \\
\text { CHLs, } \\
\text { HCB, } \\
\text { PBDEs }\end{array}$ & $\begin{array}{c}\text { PCBs: } \\
\text { Male: } 1,700 \mathrm{ng} / \mathrm{g} \\
\text { Female: } 570 \mathrm{ng} / \mathrm{g} \\
\text { DDTs: } \\
\text { Male: } 11,000 \mathrm{ng} / \mathrm{g} \\
\text { Female: } 2,300 \mathrm{ng} / \mathrm{g} \\
\text { HCHs: } \\
\text { Male: } 1,300 \mathrm{ng} / \mathrm{g} \\
\text { Female: } 210 \mathrm{ng} / \mathrm{g} \\
\text { CHLs: } \\
\text { Male: } 360 \mathrm{ng} / \mathrm{g} \\
\text { Female: } 80 \mathrm{ng} / \mathrm{g} \\
\text { HCB: } \\
\text { Male: } 130 \mathrm{ng} / \mathrm{g} \\
\text { Female: } 40 \mathrm{ng} / \mathrm{g} \\
\text { PBDEs: } \\
\text { Male: } 870 \mathrm{ng} / \mathrm{g} \\
\text { Female: } 510 \mathrm{ng} / \mathrm{g}\end{array}$ \\
\hline & & & & & & & \\
\hline [20] & $\begin{array}{l}1992- \\
2002\end{array}$ & California & $\begin{array}{l}\text { California sea otter } \\
\text { (Enhydra lutris } \\
\text { nereis) }\end{array}$ & Liver & 80 Females & PBDEs & PBDEs: 2170 ng/g \\
\hline$[7]$ & $\begin{array}{l}1988- \\
2008\end{array}$ & $\begin{array}{l}\text { England and } \\
\text { Wales }\end{array}$ & $\begin{array}{l}\text { Eurasian river otter } \\
\quad \text { (Lutra lutra) }\end{array}$ & Liver & $\begin{array}{c}129 \text { PBDE } \\
86 \text { (DDT and } \\
\text { PCB) }\end{array}$ & $\begin{array}{l}\text { PBDEs, } \\
\text { DDT, } \\
\text { PCBs }\end{array}$ & $\begin{array}{l}\text { PBDE: } 3,241.9 \mathrm{ng} / \mathrm{g} \\
\text { DDT: } 3,859 \mathrm{ng} / \mathrm{g} \\
\text { PCB: } 12,928.2 \mathrm{ng} / \mathrm{g}\end{array}$ \\
\hline & & & & & & & \\
\hline$[8]$ & $\begin{array}{l}1999- \\
2001\end{array}$ & East Greenland & $\begin{array}{l}\text { Polar bear (Ursus } \\
\text { maritimus) }\end{array}$ & Blubber & 50 Subadult & $\begin{array}{l}\text { PCBs, } \\
\text { HCH, } \\
\text { DDT, } \\
\text { CHL, } \\
\text { Mirex, } \\
\text { Dieldrin }\end{array}$ & $\begin{array}{c}\text { PCBs: } 6,470 \mathrm{ng} / \mathrm{g} \\
\text { HCH: } 198 \mathrm{ng} / \mathrm{g} \\
\text { DDT: } 462 \mathrm{ng} / \mathrm{g} \\
\text { CHL: } 2,010 \mathrm{ng} / \mathrm{g} \\
\text { Mirex: } 4.08 \mathrm{ng} / \mathrm{g} \\
\text { Dieldrin: } 218 \mathrm{ng} / \mathrm{g}\end{array}$ \\
\hline
\end{tabular}


Citation: Green A, Larson S (2016) A Review of Organochlorine Contaminants in Nearshore Marine Mammal Predators. J Environ Anal Toxicol 6: 370. doi:10.4172/2161-0525.1000370

Page 10 of 14

\begin{tabular}{|c|c|c|c|c|c|c|c|}
\hline$[8]$ & $\begin{array}{l}1999- \\
2001\end{array}$ & East Greenland & $\begin{array}{l}\text { Polar bear (Ursus } \\
\text { maritimus) }\end{array}$ & Blubber & $\begin{array}{l}25 \text { Adult } \\
\text { females }\end{array}$ & $\begin{array}{c}\text { PCBs, } \\
\text { HCH, } \\
\text { DDT, } \\
\text { CHL, } \\
\text { Mirex, } \\
\text { Dieldrin }\end{array}$ & $\begin{array}{l}\text { PCBs: } 8,240 \mathrm{ng} / \mathrm{g} \\
\text { HCH: } 263 \mathrm{ng} / \mathrm{g} \\
\text { DDT: } 462 \mathrm{ng} / \mathrm{g} \\
\text { CHL: } 2,220 \mathrm{ng} / \mathrm{g} \\
\text { Mirex: } 2.79 \mathrm{ng} / \mathrm{g} \\
\text { Dieldrin: } 208 \mathrm{ng} / \mathrm{g}\end{array}$ \\
\hline [8] & $\begin{array}{l}1999- \\
2001\end{array}$ & East Greenland & $\begin{array}{l}\text { Polar bear (Ursus } \\
\text { maritimus) }\end{array}$ & Blubber & $\begin{array}{l}16 \text { Adult } \\
\text { males }\end{array}$ & $\begin{array}{l}\text { PCBs, } \\
\text { HCH, } \\
\text { DDT, } \\
\text { CHL, } \\
\text { Mirex, } \\
\text { Dieldrin }\end{array}$ & $\begin{array}{c}\text { PCBs: } 9,100 \mathrm{ng} / \mathrm{g} \\
\text { HCH: } 218 \mathrm{ng} / \mathrm{g} \\
\text { DDT: } 559 \mathrm{ng} / \mathrm{g} \\
\text { CHL: } 1,710 \mathrm{ng} / \mathrm{g} \\
\text { Mirex: } 6.59 \mathrm{ng} / \mathrm{g} \\
\text { Dieldrin: } 245 \mathrm{ng} / \mathrm{g}\end{array}$ \\
\hline [21] & $\begin{array}{l}1999- \\
2001\end{array}$ & East Greenland & $\begin{array}{l}\text { Polar bear (Ursus } \\
\text { maritimus) }\end{array}$ & Blubber & $\begin{array}{l}52 \text { Subadult } \\
23 \text { Adult } \\
\text { females } \\
17 \text { Adult } \\
\text { males }\end{array}$ & PBDEs & $\begin{array}{c}\text { PBDE: } \\
\text { Subadult: } 68 \mathrm{ng} / \mathrm{g} \\
\text { Female: } 69 \mathrm{ng} / \mathrm{g} \\
\text { Male: } 75 \mathrm{ng} / \mathrm{g}\end{array}$ \\
\hline [69] & $\begin{array}{l}1999- \\
2001\end{array}$ & East Greenland & $\begin{array}{l}\text { Polar bear (Ursus } \\
\text { maritimus) }\end{array}$ & Liver & 20 & $\begin{array}{l}\text { PCBs, } \\
\text { PBDEs }\end{array}$ & $\begin{array}{l}\text { PCBs: } 3,125 \mathrm{ng} / \mathrm{g} \\
\text { PBDEs: } 40 \mathrm{ng} / \mathrm{g}\end{array}$ \\
\hline [69] & $\begin{array}{l}1999- \\
2001\end{array}$ & East Greenland & $\begin{array}{l}\text { Polar bear (Ursus } \\
\text { maritimus) }\end{array}$ & Blubber & 20 & $\begin{array}{l}\text { PCBs, } \\
\text { PBDEs }\end{array}$ & $\begin{array}{l}\text { PCBs: } 5,387 \mathrm{ng} / \mathrm{g} \\
\text { PBDEs: } 83 \mathrm{ng} / \mathrm{g}\end{array}$ \\
\hline [70] & 2003 & $\begin{array}{c}\text { Alaskan Beaufort } \\
\text { Sea }\end{array}$ & $\begin{array}{l}\text { Polar bear (Ursus } \\
\text { maritimus) }\end{array}$ & Blubber & 57 & $\begin{array}{l}\text { PCBs, } \\
\text { DDT, } \\
\mathrm{CHL} \text {, } \\
\mathrm{HCH}\end{array}$ & $\begin{array}{c}\text { PCBs: } 7,818 \mathrm{ng} / \mathrm{g} \\
\text { DDT: } 165 \mathrm{ng} / \mathrm{g} \\
\text { CHL: } 1,478 \mathrm{ng} / \mathrm{g} \\
\text { HCH: } 490 \mathrm{ng} / \mathrm{g}\end{array}$ \\
\hline [71] & 2007 & Hudson Bay & $\begin{array}{l}\text { Polar bear (Ursus } \\
\text { maritimus) }\end{array}$ & Blubber & 12 & $\begin{array}{l}\text { PBDEs, } \\
\text { PCBs, } \\
\text { CHL, } \\
\text { DDT, } \\
\text { Mirex }\end{array}$ & $\begin{array}{c}\text { PBDEs: } 35.4-38.8 \mathrm{ng} / \mathrm{g} \\
\text { PCB: } 2,559-3,652 \mathrm{ng} / \mathrm{g} \\
\text { CHL: } 2,697 \mathrm{ng} / \mathrm{g} \\
\text { DDT: } 105 \mathrm{ng} / \mathrm{g} \\
\text { Mirex: } 44.7 \mathrm{ng} / \mathrm{g}\end{array}$ \\
\hline
\end{tabular}

Publications by Year

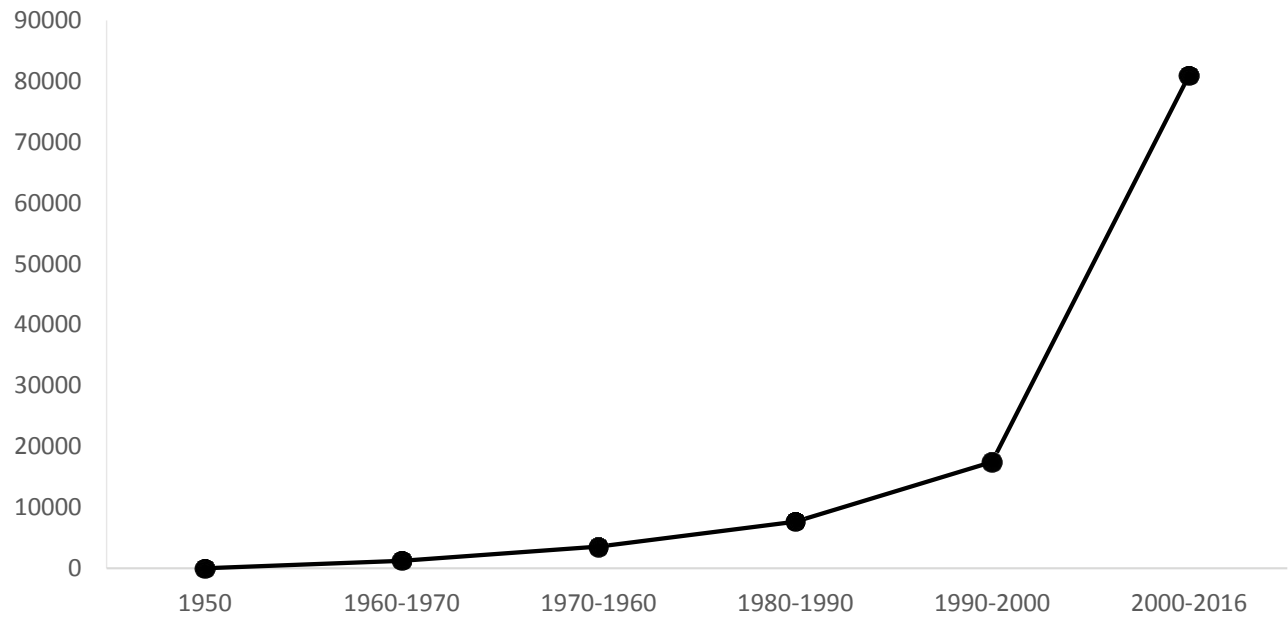

Figure 1: Significant increase in publications using keywords "toxicology of marine mammals" in Google Scholar over time. Zero papers were published in 1950, increasing to 81,000 through 2015 . 
Citation: Green A, Larson S (2016) A Review of Organochlorine Contaminants in Nearshore Marine Mammal Predators. J Environ Anal Toxicol 6: 370. doi:10.4172/2161-0525.1000370

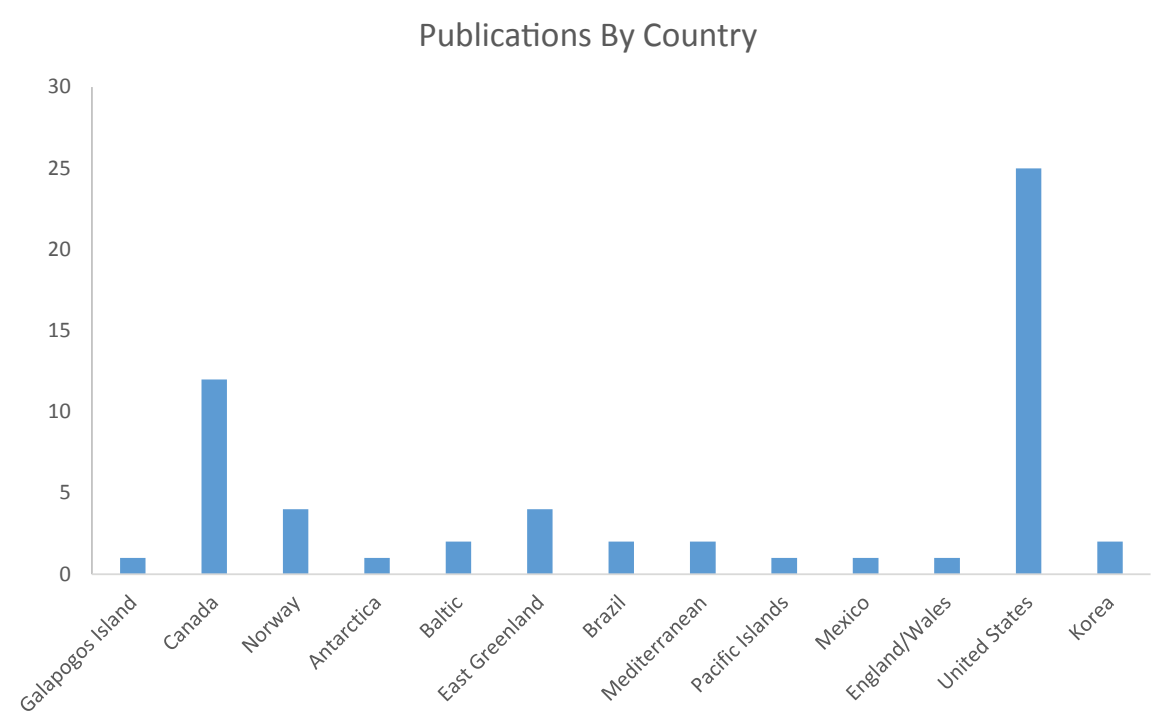

Figure 2: Marine mammal toxicology publications since 1995 and organized by country show that the majority of research is conducted in the United States followed by Canada.

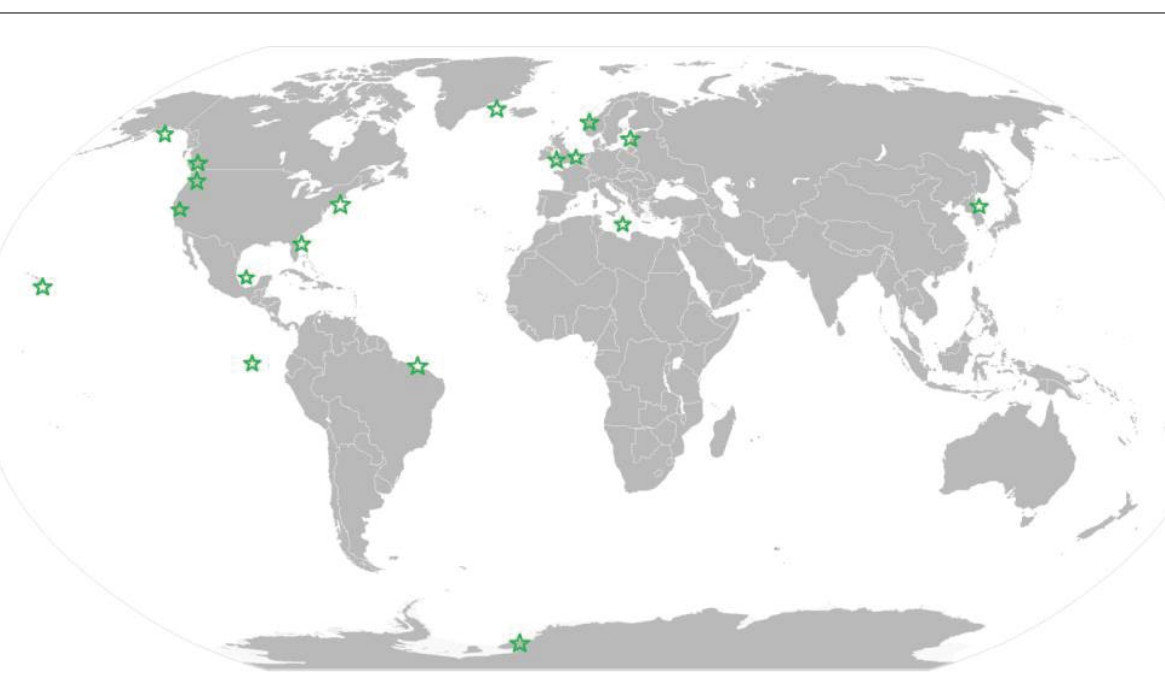

Figure 3: World map of countries that have published papers on marine mammal toxicology since 1995 show that majority of research is conducted in the Northern hemisphere.

that reported toxaphene levels are either over- or under-estimated, due to differences between sample patterns and standards. As the number and pattern of congener mixtures in the environment is much different than the technical mixtures when made, they suggest that total values given should be considered "only indicative". The LD50 of lab mammals ranged from 5 to $1075 \mathrm{mg} / \mathrm{kg}$ depending on species and route of exposure [22]. The highest toxaphene value over the last twenty years measured in pinnipeds was reported as $38 \mathrm{ng} / \mathrm{g}$ in East Greenland ringed seal blubber [23] and the highest value in cetaceans was found to be $8,206 \mathrm{ng} / \mathrm{g}$ in killer whale blubber in Norway [24]. Only two publications reported values for pinnipeds [23], and two publications were found for cetaceans [24]. All four publications collected samples either in the Pacific Island chain or between East Greenland and Norway. There were no toxaphene values given for polar bear and mustelid samples in our analysis.

Polychlorinated naphthalenes (PCNs): Polychlorinated naphthalenes (PCNs) were originally used as flame retardants and dielectric fluids for capacitors as early as 1900. Today, manufacture of PCNs is thought to have ended, although illegal importation of PCNcontaining products into Japan was reported after 2000 [25,26]. Sources to the environment include evaporation from old or in-use products containing PCNs and PCBs, and release of PCNs during combustion [27]. Polychlorinated naphthalenes are globally distributed via air, sediments, water and biologically through the food chain. They are easily transported through the atmosphere and have been found in remote areas of the arctic and subarctic regions. They are lipophilic, hydrophobic and bioaccumulate in inverts, fish, seabirds and marine mammals.

Average PCNs have been reported in a variety of arctic marine mammals: Helm et al. reported values within eastern Canadian ringed seal (Phoca hispida) of $0.045 \mathrm{ng} / \mathrm{g}$ within three males and $0.051 \mathrm{ng} / \mathrm{g}$ within three females collected in 1993 [28]. A follow up study from 
1999-2003 found much higher values of $0.33 \mathrm{ng} / \mathrm{g}$ within two males and $0.25 \mathrm{ng} / \mathrm{g}$ within 19 females [29]. Seven seals in Svalbard tested in 1981 had $0.038 \mathrm{ng} / \mathrm{g}$ [30]. Vorkam et al. reported an upper limit of $0.13 \mathrm{ng} / \mathrm{g}$ for east and west Greenland seals [31]. Wang et al. reported 39 harbor seals in gulf of Alaska (prince William sound and Kodiak island) between 2000-2001 having an average of $4.8 \mathrm{ng} / \mathrm{g}$ blubber, 1.1 $\mathrm{ng} / \mathrm{g}$ liver, and $0.59 \mathrm{ng} / \mathrm{g}$ kidney [32]. Weddell seals from terra nova bay, Antarctica had levels of $0.077 \mathrm{ng} / \mathrm{g}$ blubber and $1.63 \mathrm{ng} / \mathrm{g}$ liver [33]. Total PCN concentrations in seal blubber were positively and significantly correlated to age [34]. Canadian arctic beluga sampled in 1994 was found to be $0.33 \mathrm{ng} / \mathrm{g}$ within males and $0.18 \mathrm{ng} / \mathrm{g}$ within females [28]. While levels found in blubber of Arctic beluga collected later between 1999-2000 averaged $0.25 \mathrm{pg} / \mathrm{g}$ for males and $0.14 \mathrm{pg} / \mathrm{g}$ for females [29]. Killer whales from the northeastern pacific had levels of $21.6 \mathrm{ng} / \mathrm{g}$ within northern residents, $20.4 \mathrm{ng} / \mathrm{g}$ within southern residents and $167 \mathrm{ng} / \mathrm{g}$ within transient whales [35].

Other organochlorines: Other organochlorines contaminants have been tested in nearshore vertebrate predators and marine mammals, however data is lacking and non-comprehensive (Table 1). The following chemicals have been investigated within nearshore mammals for which there is very limited data: Mirex is an insecticide used for ants and termites, and as a flame retardant in plastics and paints. Hexachlorocyclohexan $(\mathrm{HCH})$ is an insecticide and is neurotoxic, structurally related to Mirex. Polychlorinated dibenzo-p-dioxins (PCDDs) and polychlorinated dibenzofurans (PCDFs) are by products of industrial processes. Hexachlorobenzene (HCB) is a fungicide for seed grain and is used to produce solvents and dyes. Chlordane is an organochlorine pesticide, which is now banned in Canada. It was primarily used in the 60s and 70s. Dieldrin is an organochlorine insecticide originally produced in 1948, was widely used until the 1970 s and now is banned because of its persistence in the environment.

\section{Conclusion}

There are still major limitations to our knowledge of contaminants in nearshore vertebrate predators and marine mammals. Analysis of patterns remains complex due to lack of wide scale long term results. Lab studies often expose captive animals to a single chemical at high doses for a short amount of time (acute high doses), making it difficult to extrapolate what effect low chronic doses from environmental exposure have on wildlife [36]. Free ranging wildlife is also exposed to not only one but often a complex cocktail of persistent organic pollutants. In addition uncontrolled or unknown variation in the environment (exposure to degraded mixtures that differ from original products) and the individuals such as differences in bioaccumulation potentials and excretion capabilities of nearshore vertebrate predators and marine mammals may produce vastly different physiological results compared to controlled lab studies [37]. Differences in species, age, health, sex, and proximity to human populations also complicate patterns in the data. Adding further complexity to interpreting existing data is that sampling and analytical methodology for many organochlorines is not standardized making the results not easily comparable and long term studies building on previous work challenging. An internationally standardized set of analytical methods and quality assurance procedures has yet to be developed making all existing studies using different methods and reporting in different units difficult to compare (3, Table 1). In the future it is essential that methods are standardized to ensure a better understanding of how much POPs are in wildlife and what the corresponding health and environmental effects are.

Organochlorines are manufactured and used worldwide, however there is a geographic skew in past and current research. Vos et al. draws attention to the fact that $90 \%$ of toxin samples are from the northern hemisphere, primarily Europe, Canada and the United States [38]. Although it is understood that data is geographically specific, more research is needed in varying parts of the world to analyze variance between locations and identify toxic hotspots. Since many of these compounds may travel far distances because of atmospheric deposition, it is imperative that data is collected and understood from all over the world.

Of the 36 species selected for this review (text and Table 1), three are listed as endangered on the ICUN Red List of Threatened Species. This includes the Galapagos sea lion (Zalophus wollebaeki), fin whale (Balaenoptera physalus), and California sea otter. Three species are listed as vulnerable, Franciscana dolphin (Pontoporia blainvillei), finless porpoise, and polar bear. The three species that are near threatened are Steller sea lion (Eumetopias jubatus), beluga whale, and Eurasian river otter. Many were of least concern including California sea lion (Zalophus californianus), harbor seal, ringed seal, bearded seal (Erignathus barbatus), weddell seal, Antarctic fur seal, crabeater seal (Lobodon carcinophagus), southern elephant seal (Mirounga leonine), grey seal, bottlenose dolphin (Tursiops truncates), striped dolphin (Stenella coeruleoalba), melon-headed whale (Peponocephala electra), rough toothed dolphin (Steno bredanensis), and common dolphin (Delphinus delphis). What is important to note are those species that are listed as data deficient including spotted seal (Phoca largha), ribbon seal (Histriophoca fasciata), killer whale, false killer whale (Pseudorca crassidens), pygmy killer whale (Feresa attenuate), Longman's beaked whale (Indopacetus pacificus), spinner dolphin (Stenella longirostris), dwarf sperm whale (Kogia sima), Blainville's beaked whale (Mesoplodon densirostris), Guiana dolphin (Sotalia guianensis), and long beaked common dolphin (Delphinus capensis). These data gaps only highlight the need for continued research into potential health and reproductive effects of contaminants on these species. Due to the fact that new chemicals and compounds are being synthesized every day, it is crucial not only to track legacy contaminants but also new ones within nearshore vertebrate predators including marine mammals.

\section{References}

1. Taylor BL, Baird R, Barlow J, Dawson SM, Ford J, et al. (2013) Orcinus orca The IUCN Red List of Threatened Species 2013.

2. Dierauf L, Gulland F (2001) CRC Handbook of Marine Mammal Medicine: Health, Disease, and Rehabilitation. 2nd edn. CRC Press.

3. O'Shea T (1999) Biology of Marine Mammals. Washington and London, Smithosian Institution Press, Fort Collins Science Center, pp: 485-565.

4. de Wit CA (2002) An overview of brominated flame retardants in the environment. Chemosphere 46: 583-624.

5. Ylitalo G, Matkin C, Buzitis J, Krahn M, Jones L, et al. (2001) Influence of life-history parameters on organochlorine concentrations in free-ranging killer whales (Orcinus orca) from Prince William Sound, AK. Science of the Total Environment 281: 183-203

6. Cipro CVZ, Bustamante P, Taniguchi S, Montone R (2012) Persistent organic pollutants and stable isotopes in pinnipeds from King George Island, Antarctica. Marine Pollution Bulletin 64: 2650-2655.

7. Pountney A (2008) Analysis of the Population Genetics and Polybrominated Diphenyl Ether (PBDE) Burdens of Otters in England and Wales: With Case Studies of Populations in South West England. PhD Thesis.

8. Dietz R, Riget F, Letcher S, Born W, Muir D (2004) Seasonal and temporal trends in polychlorinated biphenyls and organochlorine pesticides in Eas Greenland polar bears (Ursus maritimus), 1990-2001. Sci Total Environ 331 : 107-124. 
9. Ross PS, Couillard CM, Ikonomou MG, Johannessen SC Lebeuf M, et al. (2009) Large and growing environmental reservoirs of Deca-BDE present an emerging health risk for fish and marine mammals. Mar Pollut Bull 58: 7-10.

10. Ross P, Jeffries S, Yunker M, Addison R, Ikonomou M, et al. (2003) Harbor seals (Phoca vitulina) in British Columbia, Canada, and Washington State, USA, reveal a combination of local and global Polychlorinated Biphenyl, Dioxin and Furan signals. Environ Toxicol Chem 23: 157-165.

11. Eriksson $P$, Jakobsson $E$, Fredriksson A (2001) Brominated flame retardants: a novel class of developmental neurotoxicants in our environment? Environ Health Perspect 109: 903-908.

12. Viberg $H$, Fredriksson A, Eriksson $P$ (2002) Neonatal exposure to the brominated flame retardant 2,2,4,4,5-pentabromodiphenyl ether causes altered susceptibility in the cholinergic transmitter system in the adult mouse. Toxicol Sci 67: 104-107.

13. Zhou T, Taylor MM, DeVito MJ, Crofton KM (2002) Developmental exposure to brominated diphenyl ethers results in thyroid hormone disruption. Toxicol Sci 66: $105-116$

14. Hites RA (2004) Polybrominated diphenyl ethers in the environment and in people: a meta-analysis of concentrations. Environ Sci Technol 38: 945-956.

15. Norén K, Meironyté D (2000) Certain organochlorine and organobromine contaminants in Swedish human milk in perspective of past 20-30 years. Chemosphere 40: 1111-1123.

16. Kajiwara N, Ueno D, Takahashi A, Baba N, Tanabe S (2004) Polybrominated diphenyl ethers and organochlorines in archived northern fur seal samples from the Pacific coast of Japan, 1972-1998. Environ Sci Technol 38: 3804-3809.

17. Ramu K, Kajiwara N, Tanabe S, Lam PK, Jefferson TA (2005) Polybrominated diphenyl ethers (PBDEs) and organochlorines in small cetaceans from Hong Kong waters: levels, profiles and distribution. Mar Pollut Bull 51: 669-676.

18. Shaw SD, Berger ML, Weijs L, Covaci A (2012) Tissue-specific accumulation of polybrominated diphenyl ethers (PBDEs) including Deca-BDE and hexabromocyclododecanes (HBCDs) in harbor seals from the northwest Atlantic. Environ Int 44: 1-6.

19. Krahn MM, Hanson MB, Schorr GS, Emmons CK, Burrows DG, et al. (2009) Effects of age, sex and reproductive status on persistent organic pollutant concentrations in "Southern Resident" killer whales. Mar Pollut Bull 58: 1522-1529.

20. Kannan K, Perrotta E, Thomas NJ, Aldous KM (2007) A comparative analysis of polybrominated diphenyl ethers and polychlorinated biphenyls in Southern sea otters that died of infectious diseases and noninfectious causes. Arch Environ Contam Toxicol 53: 293-302.

21. Dietz R, Rigét FF, Sonne C, Letcher RJ, Backus S, et al. (2007) Age and seasonal variability of polybrominated diphenyl ethers in free-ranging East Greenland polar bears (Ursus maritimus). Environ Pollut 146: 166-173.

22. de Geus HJ, Besselink H, Brouwer A, Klungsøyr J, McHugh B, et al. (1999) Environmental occurrence, analysis, and toxicology of toxaphene compounds. Environ Health Perspect 107 Suppl 1: 115-144

23. Vorkamp K, Christensen JH, Riget F (2004) Polybrominated diphenyl ethers and organochlorine compounds in biota from the marine environment of East Greenland Sci Total Environ 331: 143-155.

24. Wolkers H, Corkeron PJ, Van Parijs SM, Similä T, Van Bavel B (2007) Accumulation and transfer of contaminants in killer whales (Orcinus orca) from Norway: indications for contaminant metabolism. Environ Toxicol Chem 26 : 1582-1590.

25. Falandysz J, Chudzyaski K, Takekuma M, Yamamoto T, Noma Y, et al. (2008) Multivariate analysis of identity of imported technical PCN formulation. $J$ Environ Sci Health A Tox Hazard Subst Environ Eng 43: 1381-1390.

26. Yamashita N, Taniyasu S, Hanari N, Falandysz J (2003) Polychlorinated naphthalene contamination of some recently manufactured industrial products and commercial goods in Japan. J Environ Sci Health A Tox Hazard Subs Environ Eng 38: 1745-1759.

27. Falandysz J (1998) Polychlorinated naphthalenes: an environmental update. Environ Pollut 101: 77-90.

28. Helm PA, Bidleman TF, Stern GA, Koczanski K (2002) Polychlorinated naphthalenes and coplanar polychlorinated biphenyls in beluga whale (Delphinapterus leucas) and ringed seal (Phoca hispida) from the eastern Canadian Arctic. Environ Pollut 119: 69-78.
29. Muir DCG, Alaee M, Butt C, Braune B, Helm P, et al. (2004) New contaminants in arctic biota. Synopsis of research conducted under the 2003-2004, northern contaminants program. Ottawa: Indian and Northern Affairs Canada, pp: 139-148.

30. Jansson B, Andersson R, Asplund L, Litzen K, Nylund K, et al. (1993) Chlorinated and brominated persistent organic compounds in biological samples from the environment. Environmental Toxicology and Chemistry 12: 1163-1174.

31. Vorkamp K, Dam M, Riget F, Fauser P, Bossi R, et al. (2004) Screening of "new" contaminants in the marine environment of Greenland and the Faroe Islands. NERI technical report No 525. Denmark: National Environmental Research Institute, p: 97

32. Wang D, Atkinson S, Hoover-Miller A, Li QX (2007) Polychlorinated naphthalenes and coplanar polychlorinated biphenyls in tissues of harbor seals (Phoca vitulina) from the northern Gulf of Alaska. Chemosphere 67: 2044-2057.

33. Corsolini S, Kannan K, Imagawa T, Focardi S, Giesy JP (2002) Polychloronaphthalenes and other dioxin-like compounds in Arctic and Antarctic marine food webs. Environ Sci Technol 36: 3490-3496.

34. Bidleman TF, Helm PA, Braune BM, Gabrielsen GW (2010) Polychlorinated naphthalenes in polar environments--a review. Sci Total Environ 408: 2919-2935

35. Rayne S, Ikonomou MG, Ross PS, Ellis GM, Barrett-Lennard LG (2004) PBDEs, PBBs and PCNs in three communities of free-ranging killer whale (Orcinus orca) from the northeastern Pacific Ocean. International Environ Sci Technol 38: 4293-4299.

36. Letcher RJ, Bustnes JO, Dietz R, Jenssen BM, Jørgensen EH, et al. (2010) Exposure and effects assessment of persistent organohalogen contaminants in arctic wildlife and fish. Sci Total Environ 408: 2995-3043.

37. Ross $P$ (2000) Marine Mammals as Sentinels in Ecological Risk Assessment. Human and Ecological Risk Assessment 6: 29-46.

38. Vos JG, Bossart G, Fournier M, O'Shea T (2003) Toxicology of Marine Mammals. Taylor \& Francis, CRC Press.

39. Kajiwara N, Kannan K, Muraoka M, Watanabe M, Takahashi S, et al. (2001) Organochlorine pesticides, polychlorinated biphenyls, and butyltin compounds in blubber and livers of stranded California sea lions, elephant seals, and harbor seals from coastal California, USA. Arch Environ Contam Toxicol 41: 90-99.

40. Alava JJ, Ikonomou MG, Ross PS, Costa D, Salazar S, et al. (2009) Polychlorinated biphenyls and polybrominated diphenyl ethers in Galapagos sea lions (Zalophus wollebaeki). Environ Toxicol Chem 28: 2271-2282.

41. Alava JJ, Lambourn D, Olesiuk P, Lance M, Jeffries SJ, et al. (2012) PBDE flame retardants and PCBs in migrating Steller sea lions (Eumetopias jubatus) in the Strait of Georgia, British Columbia, Canada. Chemosphere 88: 855-864.

42. Kelly BC, Ikonomou MG, Blair JD, Gobas FAPC (2008) Hydroxylated and Methoxylated Polybrominated Diphenyl Ethers in a Canadian Arctic Marine Food Web. Environmental Science \& Technology 42: 7069-7077.

43. Neale JC, Gulland FM, Schmelzer KR, Harvey JT, Berg EA, et al. (2005) Contaminant loads and hematological correlates in the harbor seal (Phoca vitulina) of San Francisco Bay, California. J Toxicol Environ Health A 68: 617-633.

44. Stapleton HM, Dodder NG, Kucklick JR, Reddy CM, Schantz MM, et al (2006) Determination of HBCD, PBDEs and MeO-BDEs in California sea lions (Zalophus californianus) stranded between 1993 and 2003. Mar Pollut Bull 52 522-531.

45. Quakenbush LT (2007) Polybrominated diphenyl ether compounds in ringed, bearded, spotted, and ribbon seals from the Alaskan Bering Sea. Marine Pollution Bulletin 54: 226-246.

46. She J, Petreas M, Winkler J, Visita P, McKinney M, et al. (2002) PBDEs in the San Francisco Bay Area: measurements in harbor seal blubber and human breast adipose tissue. Chemosphere 46: 697-707.

47. Ross $P$, Noel M, Lambourn D, Dangerfield N, Calambokidis J, et al. (2013) Declining concentrations of persistent PCBs, PBDEs, PCDEs, and PCNs in harbor seals (Phoca vitulina) from the Salish Sea. Progress in Oceanography 115: $160-170$.

48. Noel M, Ross P, Jeffries S, Lance M (2011) Toxic Contaminants in Harbor Seal (Phoca vitulina) Pups from Puget Sound. Washington Department of Fish and Wildlife, pp: 1-62.

49. Routti H, Lydersen C, Hanssen L, Kovacs KM (2014) Contaminant levels in the world's northernmost harbor seals (Phoca vitulina). Mar Pollut Bull 87: 140-146. 
Citation: Green A, Larson S (2016) A Review of Organochlorine Contaminants in Nearshore Marine Mammal Predators. J Environ Anal Toxicol 6: 370. doi:10.4172/2161-0525.1000370

50. Routti H, van Bavel B, Letcher RJ, Arukwe A, Chu S, et al. (2009) Concentrations, patterns and metabolites of organochlorine pesticides in relation to xenobiotic phase I and II enzyme activities in ringed seals (Phoca hispida) from Svalbard and the Baltic Sea. Environ Pollut 157: 2428-2434.

51. Routti H, Nyman M, Jenssen BM, Bäckman C, Koistinen J, et al. (2008) Bonerelated effects of contaminants in seals may be associated with vitamin $D$ and thyroid hormones. Environ Toxicol Chem 27: 873-880.

52. Letcher R, Gebbink W, Sonne C, Born E (2009) Bioaccumulation and biotransformation of brominated and chlorinated contaminants and their metabolites in ringed seals (Pusa hispida) and polar bears (Ursus maritimus) from East Greenland. Environ Int 35: 1118-1124.

53. Rigét F, Vorkamp K, Dietz R, Rastogi SC (2006) Temporal trend studies on polybrominated diphenyl ethers (PBDEs) and polychlorinated biphenyls (PCBs) in ringed seals from east Greenland. J Environ Monit 8: 1000-1005.

54. Shaw SD, Brenner D, Berger ML, Fang F, Hong CS, et al. (2008) Bioaccumulation of polybrominated diphenyl ethers in harbor seals from the northwest Atlantic. Chemosphere 73: 1773-1780.

55. Alonso MB, Eljarrat E, Gorga M, Secchi ER, Bassoi M, et al. (2012) Natural and anthropogenically-produced brominated compounds in endemic dolphins from Western South Atlantic: another risk to a vulnerable species. Environ Pollut 170: 152-160.

56. Fair PA, Mitchum G, Hulsey TC, Adams J, Zolman E, et al. (2007) Polybrominated diphenyl ethers (PBDEs) in blubber of free-ranging bottlenose dolphins (Tursiops truncatus) from two southeast Atlantic estuarine areas. Arch Environ Contam Toxicol 53: 483-494.

57. Aguilar A, Borrell A (2005) DDT and PCB reduction in the western Mediterranean from 1987 to 2002, as shown by levels in striped dolphins (Stenella coeruleoalba). Mar Environ Res 59: 391-404.

58. Bachman MJ, Keller JM, West KL, Jensen BA (2014) Persistent organic pollutant concentrations in blubber of 16 species of cetaceans stranded in the Pacific Islands from 1997 through 2011. Sci Total Environ 488-489: 115-23.

59. Dorneles PR, Sanz P, Eppe G, Azevedo AF, Bertozzi CP, et al. (2013) High accumulation of PCDD, PCDF, and PCB congeners in marine mammals from Brazil: a serious PCB problem. Sci Total Environ 463-464: 309-18.

60. Fossi MC, Casini S, Marsili L, Ancora S, Mori G, et al. (2002) Biomarkers of Exposure and Effects for Assessing Toxicological Risk of Endocrine Distrupters in Top Predators of the Mediterranean Sea. Marine Ecology 23: 184-189.
61. Krahn MM, Hanson MB, Baird RW, Boyer RH, Burrows DG, et al. (2007) Persistent organic pollutants and stable isotopes in biopsy samples (2004/2006) from Southern Resident killer whales. Mar Pollut Bull 54: 1903-1911.

62. Andersen G, Kovacs KM, Lydersen C, Skaare JU, Gjertz I, et al. (2001) Concentrations and patterns of organochlorine contaminants in white whales (Delphinapterus leucas) from Svalbard, Norway. Sci Total Environ 264: 267-281.

63. McKinney M, De Guise S, Martineau D, Beland $P$, Lebeuf $M$, et al (2006) Organohalogen Contaminants and Metabolites in Beluga Whale (Delphinapterus leucas) Liver from Two Canadian Populations. Environmental Toxicology and Chemistry 25: 30-41.

64. Johnson-Restrepo B, Kannan K, Addink R, Adams D (2005) Polybrominated Diphenyl Ethers and Polychlorinated Biphenyls in a Marine Foodweb of Coastal Florida. Envion Sci Technol 39: 8243-8250.

65. Litz JA, Garrison LP, Fieber LA, Martinez A, Contillo JP, et al. (2007) Finescale spatial variation of persistent organic pollutants in bottlenose dolphins (Tursiops truncatus) in Biscayne Bay, Florida. Environ Sci Technol 41: 7222 7228.

66. Moon HB, Kannan K, Choi M, Yu J, Choi HG, et al. (2010) Chlorinated and brominated contaminants including PCBs and PBDEs in minke whales and common dolphins from Korean coastal waters. J Hazard Mater 179: 735-741.

67. Herman D, Burrows D, Wade P, Durban J, Matkin C, et al. (2005) Feeding ecology of eastern North Pacific killer whales Orcinus orca from fatty acid, stable isotope, and organochlorine analysis of blubber biopsies. Mar Ecol Prog Ser 302: 275-291.

68. Park BK, Park GJ, An YR, Choi HG, Kim GB, et al. (2010) Organohalogen contaminants in finless porpoises (Neophocaena phocaenoides) from Korean coast waters: Contamination status, maternal transfer and ecotoxicological implications. Mar Pollut Bull 60: 768-774.

69. Gebbink WA, Sonne C, Dietz R, Kirkegaard M, Riget FF, et al. (2008) Tissuespecific congener composition of organohalogen and metabolite contaminants in East Greenland polar bears (Ursus maritimus). Environ Pollut 152: 621-629.

70. Bentzen TW, Muir DC, Amstrup SC, O'Hara TM (2008) Organohalogen concentrations in blood and adipose tissue of Southern Beaufort Sea pola bears. Sci Total Environ 406: 352-367.

71. McKinney MA, Stirling I, Lunn NJ, Peacock E, Letcher RJ (2010) The role of diet on long-term concentration and pattern trends of brominated and chlorinated contaminants in western Hudson Bay polar bears, 1991-2007. Sc Total Environ 408: 6210-6222. 